\title{
Dynamics of Laser Produced Plasma From Foam Targets for Future Nanolithography Devices and X- Ray Sources
}

\section{Yaoxing Wu}

Huazhong University of Science and Technology

\section{Girik Jain}

Purdue University

\section{Tatyana Sizyuk}

Purdue University

\section{Xinbing Wang}

Huazhong University of Science and Technology

Ahmed Hassanein ( $\nabla$ hassanein@purdue.edu )

Purdue University

\section{Research Article}

Keywords: low density foam targets, laser produced plasma, ion dynamics, plasma expansion, mass ablation rate

Posted Date: May 14th, 2021

DOl: https://doi.org/10.21203/rs.3.rs-505829/v1

License: (c) (i) This work is licensed under a Creative Commons Attribution 4.0 International License. Read Full License 
3 Yaoxing $\mathrm{Wu}^{1,2}$, Girik Jain ${ }^{2}$, Tatyana Sizyuk ${ }^{2}$, Xinbing Wang ${ }^{1,}$, , Ahmed Hassanein ${ }^{2, *}$

$4{ }^{1}$ Wuhan National Laboratory for Optoelectronics, Huazhong University of Science and Technology,

5 Wuhan 430074, China

$6{ }^{2}$ Center for Materials Under Extreme Environment (CMUXE), Purdue University, West Lafayette,

7 Indiana 47907, USA

8 Email addresses: Yaoxing Wu (yaoxingwu@hust.edu.cn), Girik Jain (jain311@purdue.edu),

9 Tatyana Sizyuk (tsizyuk@purdue.edu), Xinbing Wang (xbwang@hust.edu.cn), Ahmed Hassanein

10 (hassanein@purdue.edu)

$11 *$ Corresponding author: Ahmed Hassanein (Tel: 765-496-9731, Fax: 765-496-2233) Xinbing Wang (Tel: 18986129186, Fax: 02787792355 )

\section{Abstract:}

Foam targets are expected to be more efficient candidates than solid targets for laser produced plasma (LPP) for extreme ultraviolet (EUV) and x-ray radiation sources due to the expected plasma conditions that can be optimized regarding plasma opacities, volumetrics heating effects, and the produced ions debris characteristics. In this paper, a comparison of ion dynamics between low-density foam and solid Ni plasma was systematically investigated at CMUXE. The foam Ni target (density $0.6 \mathrm{~g} / \mathrm{cm}^{3}$ ) and solid Ni target (density $8.9 \mathrm{~g} / \mathrm{cm}^{3}$ ) were irradiated with $1064 \mathrm{~nm} \mathrm{Nd:YAG} \mathrm{laser} \mathrm{in}$ vacuum. A Faraday cup (FC) was used to record the ion flux and time-of-flight (TOF) signals. A lower and wider TOF signal was observed for foam Ni plasma on the time scale. The average ion energy and peak of the TOF signal of solid Ni plasma were much higher than that of the foam Ni plasma. However, the total charge values between foam and solid Ni plasma were comparable indicating a more volumetric absorption of laser energy for foam Ni. The average ion energy and peak of the TOF signal of solid $\mathrm{Ni}$ 
showed a stronger angular and laser energy dependence than that of foam $\mathrm{Ni}$. The plume shape of the solid Ni plasma appeared as an oblong ellipse at each time, while that of foam Ni plasma tended to be more circular, especially at early times. The results of mass ablation rate were consistent with the FC signals and showed a more intense plasma shielding for solid $\mathrm{Ni}$.

Keywords: low density foam targets; laser produced plasma; ion dynamics; plasma expansion; mass ablation rate

\section{Introduction}

Laser produced plasma (LPP) with a high-power laser pulse opens up a new path for the development of innovative extreme ultraviolet (EUV) and x-ray sources which are very important for wide-ranging applications. These include advanced lithography ${ }^{1}$, x-ray backlighting ${ }^{2}$, inertial confinement fusion (ICF) ${ }^{3}$, Thomson scattering ${ }^{4}$, high energy density physics (HEDP) ${ }^{5}$, radiography ${ }^{6}$, etc. In these applications, it is crucial to acquire the EUV and x-ray sources with a high brightness and high conversion efficiency (CE) of laser to desired EUV or x-ray radiation output. The EUV and x-ray yield is determined by the plasma state which is significantly dependent on irradiation conditions such as laser intensity, pulse duration, focusing spot size, waveform, laser wavelength, etc. ${ }^{7-12}$. Furthermore, the target parameters (atomic number, structure, density, geometric shape, thermal conductivity, heat capacity, etc.) will also influence the final $\mathrm{CE}$ and an efficient source can be achieved by optimizing these parameters ${ }^{13-}$ ${ }^{18}$. Currently, one important issue for the interaction between laser and solid targets is that the latter is highly opaque, thus limiting the interaction region to the skin layer of the target surface. Furthermore, the electron density of the plasma produced by the leading edge of the laser will reach the critical density quickly because of the highdensity of the solid target, which causes a significant fraction of the laser energy to be reflected and a rather low laser absorption rate ${ }^{19,20}$. However, reducing the initial 
57 density of target used in LPP could effectively solve this issue ${ }^{21}$. Recently, the interaction between the laser and low-density foam targets is becoming a research hotspot.

When a pulsed laser is incident upon a low-density foam target, the laser is able to penetrate and heat the target directly to a much greater depth/volume and a controlled, low-density plasma will be generated, showing the so-called volumetric heating effect and relatively uniform density and temperature compared with solid targets ${ }^{22}$. These characteristics of foam targets can increase the laser absorption rate and reduce the ion kinetic energy, which is very beneficial for the EUV or x-ray conversion devices ${ }^{23}$. In the field of semiconductor lithography, liquid tin droplets irradiated by a double pulse laser scheme is currently adopted to enhance the EUV emission intensity with $100 \mathrm{kHz}$ repetition ${ }^{24,25}$. However, the double pulse scheme has a durability problem due to the difficulty of controlling the droplet expansion dynamics and fragmentation caused by the pre-pulse ${ }^{22,26}$. Currently, some research related to the laser produced low-density tin plasma show that the complex double pulse laser scheme is expected to be replaced by using low-density foam targets in the future ${ }^{26-28}$. In addition, low density foam targets have been demonstrated to have higher $\mathrm{x}$-ray radiation than solid targets in reports of the laser produced $\mathrm{Au}^{23,29,30}, \mathrm{Cu}^{31}, \mathrm{~V}^{31}, \mathrm{Ti}^{32}, \mathrm{Ca}^{33}, \mathrm{Bi}^{34}$, and cellulose triacetate ${ }^{20}$ plasma. Thus, low density foam targets are expected to be the most suitable candidates for the efficient LPP EUV and x-ray sources ${ }^{20}$.

Currently, most of studies related to low density foam targets in LPP focus on their EUV or x-ray radiation. Previously, Harilal et al. ${ }^{28}$ compared the EUV spectral purity and ion debris mitigation between the low-density and solid Sn targets by using a transverse magnetic field. The results show that the ion flux can be effectively reduced 81 more than 1 order of magnitude for the low-density Sn targets by using a $0.64 \mathrm{~T}$ 82 magnetic field, while the magnetic field slowed down only Sn ions for solid Sn plasma. 83 The unresolved transition array (UTA) spectrum at $13.5 \mathrm{~nm}$ showed a narrower distribution for low-density Sn plasma. Yunsong et al. ${ }^{30}$ studied the detailed energy 
distributions in laser produced solid and foam gold plasma by using a 1D hydrodynamic code. The calculated results show that the foam gold targets have stronger x-ray radiation and lower kinetic energy of ions, which is ascribed to the larger x-ray emission zone and smaller conduction zone. Fazeli ${ }^{33}$ simulated the effects of target porosity on the x-ray line emission from laser produced Ca plasma in the water-window region for different laser pulses with different intensities and durations. The results calculated with 1D Lagrangian laser plasma code also indicated that the x-ray lines were enhanced considerably for low-density $\mathrm{Ca}$ targets and the plasma ion density can be turned by increasing the target porosity. Lu et al. ${ }^{35}$ reported the plasma expansion with gold foam irradiated by laser. The theoretical analysis and MULTI 1D simulation results show that the density contour of gold foam moved slower than that of solid gold. Kaur et al. ${ }^{20,29,36}$ investigated the interaction of high-power sub nanosecond laser pulses with lowdensity cellulose triacetate polymer and gold foam targets. They demonstrated that the low-density foam targets in LPP could enhance the x-ray yield and reduce the kinetic energy of ions. Although many papers show that the x-ray radiation could be enhanced by using low density foam targets, further studies are still needed to fully understand the physics of laser interaction of low-density foam targets. Furthermore, although some papers report a decrease in ion kinetic energy for the low-density foam targets, the majority of these studies are theoretically calculated results obtained through some simulations. Per our knowledge, there are very few experimental studies on the comparison of ion dynamics, plasma plume expansion and mass ablation rate between the solid targets and low-density foam targets. It is well known that pulsed LPP is a transient process, and the plasma state will evolve rapidly with time and space. It is essential to obtain information about laser energy absorption, heat transfer, electronlattice energy exchange, plasma plume formation and expansion, material melting and evaporation, etc. The determination of the ion dynamics, plasma plume expansion evolved with time and space and mass ablation rate dependence on laser energy could result in better understanding of the physics of laser interaction with low-density foam 
targets, which is critical for efficient LPP EUV and x-ray sources.

In this paper, we have systematically studied and compared the characteristics of laser produced solid and low-density foam Ni plasma. The ions emitted from the LPP were collected by a Faraday cup (FC). The total charge, time-of-flight (TOF) signals and ion kinetic energy distributions of the LPP for solid and foam Ni plasma have been studied and compared in detail. In particular, the influence of laser intensity on these parameters and their angular dependence was also investigated. An intensified charge coupled device (ICCD) was used to capture the two-dimensional plasma plume images and a microbalance was adopted to calculate the mass ablation rate of the different targets. These experimental results provide more insight into the basic processes of ion emission, ion kinetic energy distributions, plume expansion dynamics, and the interaction between the laser and target.

\section{Experimental setup}

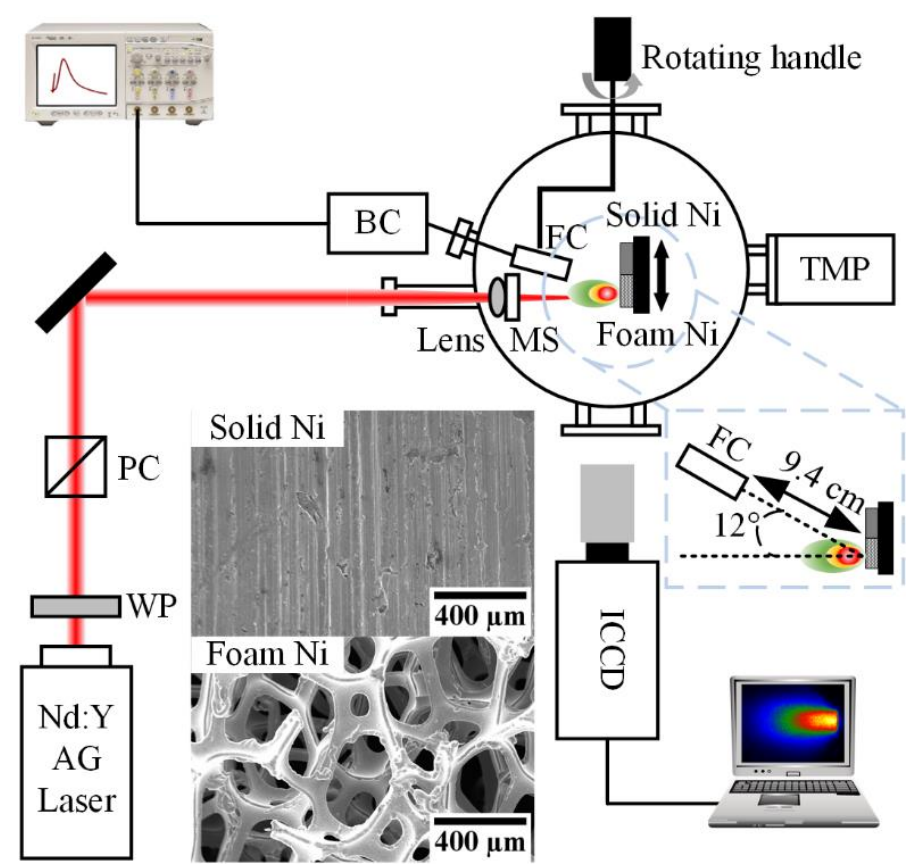

Figure 1 The schematic of the experimental setup in CMUXE and the pictures of solid and foam Ni under SEM. BC, biasing circuit; FC, Faraday cup; MS, microscope slide, TMP, turbomolecular pump; PC, polarizing cube; WP, half waveplate; ICCD, intensified charged coupled device.

The schematic of the experimental setup in CMUXE is shown in Figure 1. A Qswitched Nd:YAG (Continuum Surelite III) laser was used for generating plasma in a vacuum chamber and operated at the wavelength of $1064 \mathrm{~nm}$. The full width half 
maximum (FWHM) pulse duration and unfocused circular beam diameter were $6 \mathrm{~ns}$ and $10 \mathrm{~mm}$, respectively. A combination of a half waveplate and a polarizing cube was used to attenuate the output laser pulse energies. The laser energies were adjusted from $100 \mathrm{~mJ}$ to $400 \mathrm{~mJ}$ in steps of $25 \mathrm{~mJ}$ to investigate the dependence of laser energy on ion dynamics. A $20 \mathrm{~cm}$ plano-convex lens was adopted to focus the laser beam onto the surface of the planar solid and foam Ni targets. A very thin microscope slide was connected to the lens to prevent the contamination coming from plasma debris and would be replaced after each 1000 pulses. The targets were placed in front of the lens focal point to obtain a larger spot size which was $520 \mu \mathrm{m}$. The peak power density of the laser for $100 \mathrm{~mJ}, 200 \mathrm{~mJ}, 300 \mathrm{~mJ}$, and $400 \mathrm{~mJ}$ was $7.82 \times 10^{9} \mathrm{~W} / \mathrm{cm}^{2}, 1.56 \times 10^{10}$ $\mathrm{W} / \mathrm{cm}^{2}, 2.35 \times 10^{10} \mathrm{~W} / \mathrm{cm}^{2}$, and $3.13 \times 10^{10} \mathrm{~W} / \mathrm{cm}^{2}$, respectively.

The densities of the solid and foam Ni target were $8.9 \mathrm{~g} / \mathrm{cm}^{3}$ and $0.6 \mathrm{~g} / \mathrm{cm}^{3}$, with purities of $99.96 \%$ and $99.8 \%$, respectively. The foam Ni target showed an open-cell network structure under a scanning electron microscope (SEM) which is shown in Figure 1. Both of the solid and foam Ni were mounted side by side to a remotely controlled servomotor (ThorLabs Z825B) XYZ translation stage, which translated the targets around the laser irradiating position to provide fresh surface exposure for each measurement. The position of laser focal point could also be adjusted on different areas of the target surface by the servomotor without opening the vacuum chamber, making sure that the experimental results of solid and foam Ni were obtained at the same conditions. The pressure in the vacuum chamber was kept at $\sim 2 \times 10^{-5}$ Torr during the experiment to minimize the charge exchange with the residual ambient gas.

A cylindrical Faraday cup (FC) ion collector (IC) with a $3 \mathrm{~mm}$ entrance aperture diameter was placed at a distance of $9.4 \mathrm{~cm}$ from the target surface to monitor the flux and kinetic energy of the ions induced by the laser-target interaction. The Faraday cup was mounted on a rotating handle with an angle meter which allowed the angle of measurement to be changed from outside the chamber. When the angle meter on the rotating handle showed $0^{\circ}$, it meant that the $\mathrm{FC}$ and the laser spot were on the same 
161 horizontal plane. At the time illustrated in Figure 1, the angle between the FC and the

162 target normal was $12^{\circ}$. A bias voltage of $-30 \mathrm{~V}$ was applied to collect the ions and to 163 repel the electrons emitted from the plasma. The output signal was monitored and 164 recorded using a $50 \Omega$ load resistor $1 \mathrm{GHz}$ oscilloscope (Agilent infiniium MSO8104A).

165 Each ion signal was obtained with same experimental conditions by averaging the 166 voltage signals 10 shots to minimize the influence of the fluctuation in the shot-to-shot 167 laser energy.

168 For the evolution of plasma plumes, a series of time resolved plasma images were 169 captured by an intensified charged coupled device (ICCD) camera (Andor iStar 170 DH334T-18U-E3 1024×1024) using a tele-photo objective lens (Edmund Optics 75 mm

171 DG Series Fixed Focal Length Lens) through a quartz window in the vacuum chamber 172 at a $90^{\circ}$ angle with respect to the incident laser. The plasma images were composed of 173 integrated radiation from a wavelength region of $180-850 \mathrm{~nm}$. The gate width with $5 \mathrm{~ns}$ 174 and $10 \mathrm{~ns}$ were used to image plasma at early times $(<100 \mathrm{~ns})$ and at later times, 175 respectively.

176 A microbalance (METTLER TOLEDO XS3DU, dual range balance with $1 \mu \mathrm{g}$ 177 readability) was used to measure the target mass before and after being irradiated by 178 the pulsed laser. Two hundred shots were accumulated at different laser energies and 179 the mass difference was used to calculate the mass ablation rate. All experiments were 180 repeated 5 times and then take an average as the final results to improve the reliability 181 of the data.

\section{3. Results and discussion}

183 3.1 Ion measurements

184 Understanding the ion emission characteristics and the effect of laser intensity on 185 the ions' kinetic energy and angular dependence are essential to investigate LPP 186 evolution and dynamics. 

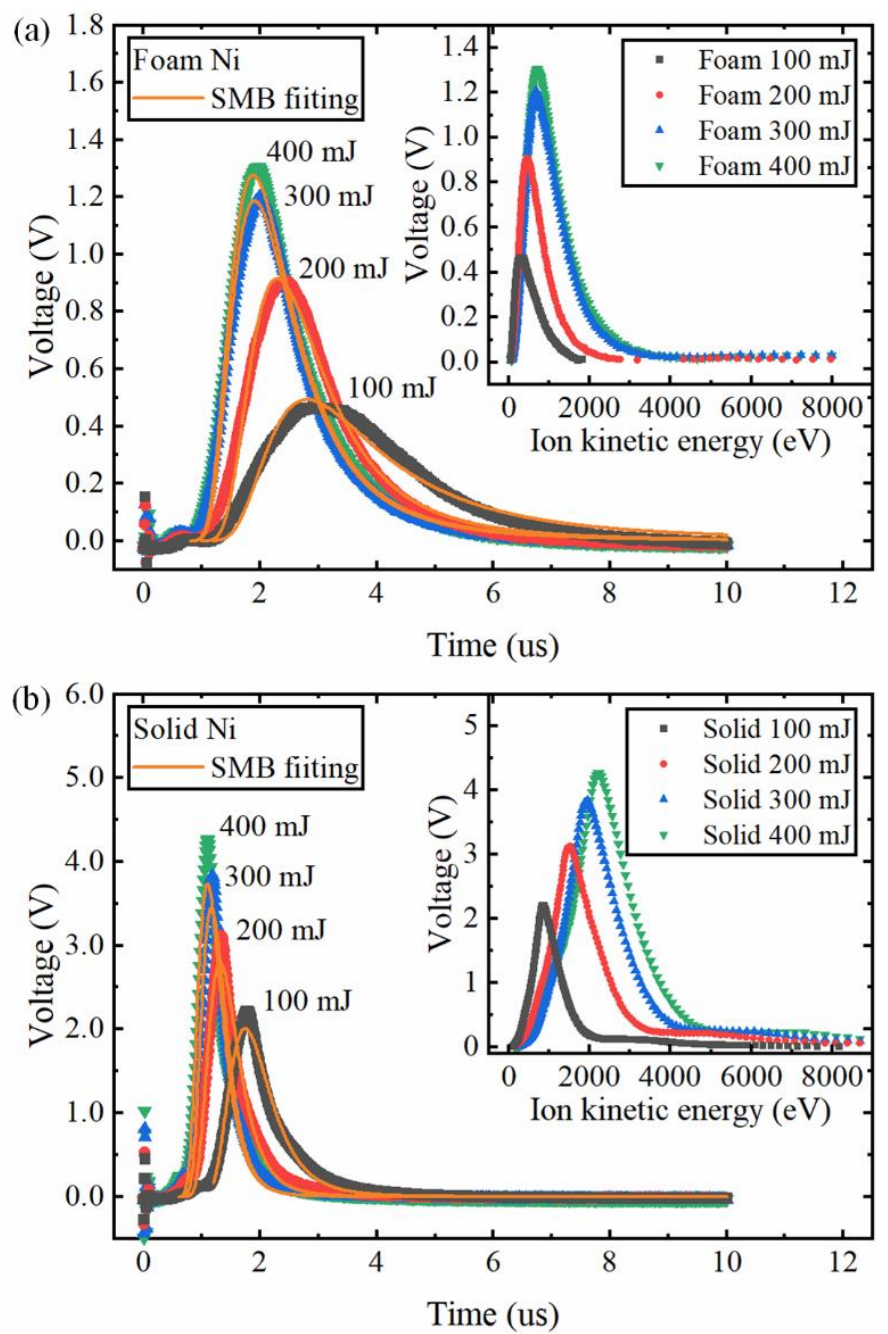

Figure 2 Typical ion time-of-flight (TOF) signal obtained from the Faraday cup ion collector for various laser energies for (a) Foam Ni and (b) Solid Ni. The Shifted Maxwell Boltzmann distribution was used to fit the thermal peaks. The inset shows the ion kinetic energy distribution converted from the TOF signal.

Figure 2 shows a typical TOF signal obtained from the Faraday cup ion collector

193 for various laser energies. Each curve was the average of the TOF signal for 10

194 consecutive laser shots. In general, the TOF signal of the foam and solid Ni all reached a peak quickly with time under different laser energies, and then decreased as the plume continued to expand beyond the FC. In addition, the rising rate of the TOF signal and

197 its peak increased and the position of ion peak shifted to earlier times as the laser energy

198 increased, indicating an enhancement in the number of high-energy ions. In nanosecond

199 LPP, the electrostatic model ${ }^{37}$ is usually used to explain the ion acceleration. The 200 electrons will absorb the laser energy via inverse bremsstrahlung (IB) process and 201 transfer the absorbed laser energy to ions through electron-ion collisions on the time 
scale of the electron-ion thermalization time $\left(10^{-10}-10^{-11} \mathrm{~s}\right)$ which is much shorter than

203 the laser pulse duration. In other words, the electrons and ions reach equal thermal 204 energies at the end of the laser. However, the electron velocities are much higher than 205 that of the ions due to the huge mass difference. A space charge separation in the plasma 206 plume is formed due to the huge velocity difference between the energetic electrons and 207 the heavy ions, generating a self-electrostatic field. The ions are accelerated in this self208 electrostatic field and the final ion kinetic energy is proportional to their charge state. 209 However, this ion acceleration due to the generation of electrostatic field is more 210 pronounced at higher laser intensities. Figure 2 shows such high energy ions detected 211 at earlier time which are small fraction of the total ions kinetic energy. Previous 212 experiments showed two peaks of ion kinetic energies in LPPs related to small fast ions 213 fraction and large slow ions fraction ${ }^{38}$. HEIGHTS 3D simulations predicted very 214 accurately the kinetic energies of the large ion fraction showing that most of kinetic 215 ions in LPPs at the considered laser intensities can be explained by hydrodynamic 216 effects in quasi neutral plasma plume ${ }^{8,39}$. Therefore, the increase of the average ion 217 charge state as well as increasing plasma kinetic energy with the increase of laser energy 218 is the most probably reason for the increase in ion kinetic energy of solid and foam $\mathrm{Ni}$ 219 plasma. The increasing difference of average ion energy between solid and foam $\mathrm{Ni}$ 220 plasma is shown in Figure 3(a) and will be discussed later.

221 Generally, the LPP will experience complicated processes of ionization and 222 recombination after it is generated. The TOF signal obtained from Faraday cup ion 223 collector is composed of all ion energies and charge states emitted from LPP. Under the 224 combination of ionization and recombination process, a shifted Maxwell-Boltzmann 225 (SMB) is usually used to describe the ion kinetic energy distribution in LPP ${ }^{37}$ :

$$
F(t)=C\left(\frac{L}{t}\right)^{3} \exp \left[-\frac{m\left(\frac{L}{t}-v_{f i t}\right)^{2}}{2 k_{B} T_{x}}\right]
$$

227 where $C$ is a scale parameter, $L$ is the distance between the FC and target surface, 
$v_{f i t}$ is the center-of-mass velocity which is determined by the adiabatic expansion and

229 the coulomb acceleration, $k_{B}$ is the Boltzmann constant, and $T_{x}$ is the translational 230 temperature. According to the SMB fitting, the center-of-mass velocity of foam $\mathrm{Ni}$ 231 plasma at $100 \mathrm{~mJ}, 200 \mathrm{~mJ}, 300 \mathrm{~mJ}$, and $400 \mathrm{~mJ}$ were $1.62 \times 10^{4} \mathrm{~m} / \mathrm{s}, 2.88 \times 10^{4} \mathrm{~m} / \mathrm{s}$, $2323.39 \times 10^{4} \mathrm{~m} / \mathrm{s}, 3.40 \times 10^{4} \mathrm{~m} / \mathrm{s}$, and that of solid Ni plasma were $4.66 \times 10^{4} \mathrm{~m} / \mathrm{s}, 6.22 \times 10^{4}$ $233 \mathrm{~m} / \mathrm{s}, 7.22 \times 10^{4} \mathrm{~m} / \mathrm{s}$, and $7.55 \times 10^{4} \mathrm{~m} / \mathrm{s}$, respectively. These velocities provide guiding 234 relevance for the plasma plume expansion. Based on the comparison of center-of-mass 235 velocity between the foam and solid Ni plasma, we can also infer that the plasma plume 236 expanded faster for solid Ni plasma along the target normal, which was also verified by 237 the time-resolved plasma plume images in next section.

238 The shape of TOF signal highly depends on the ion density distribution in the 239 plasma plume along the specific direction. The TOF signal of foam Ni plasma in Figure 2402 experienced a longer time and showed a much smaller amplitude than that of solid $\mathrm{Ni}$ 241 plasma, indicating a lower and wider distribution for foam Ni plasma on the time scale. 242 This meant that the ion density distribution in the foam Ni plasma plume was relatively 243 more uniform compared to solid Ni plasma. Similar results are found in laser produced 244 low-density polymer aerogels plasma ${ }^{36}$ and gold foams plasma ${ }^{29}$ studies. The inset in 245 Figure 2 shows the ion kinetic energy distribution converted from the TOF signal which 246 provide a more intuitive comparison of the ion kinetic energy between the solid and 247 foam Ni plasma. The proportion of high-kinetic energy ions in the solid Ni plasma was 248 much higher than that of the foam Ni plasma. For example, in the case of the $400 \mathrm{~mJ}$ 249 laser energy, there were almost no ions with kinetic energy greater than $2000 \mathrm{eV}$ in the 250 foam Ni plasma, while nearly half of the ions with kinetic energy greater than this value 251 existed in the solid Ni plasma. This is very important in terms of the lifetime of the 252 optical collecting system, e.g., in nanolithography devices. Lower energy ions of the 253 foam targets will cause less damage than the energetic ions of the solid targets. 

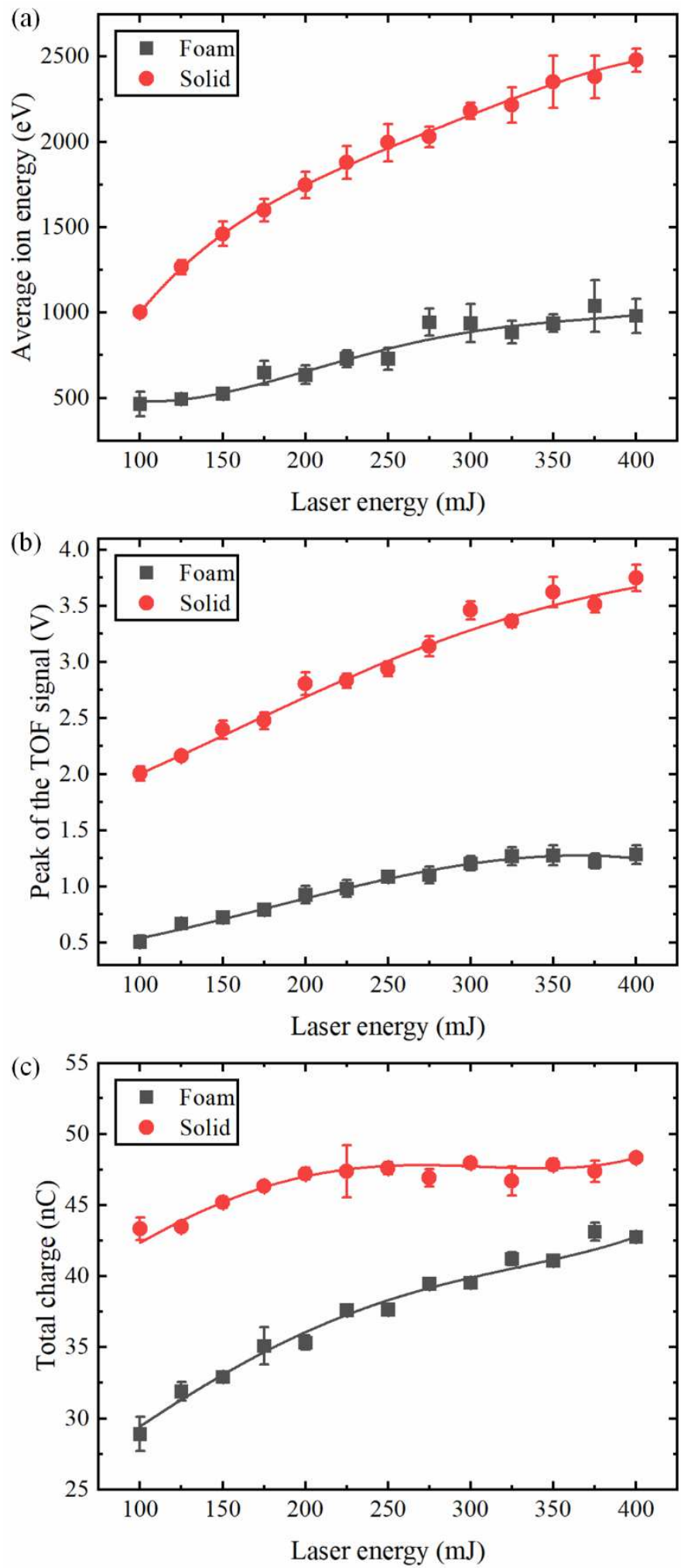

255 Figure 3 Effect of the laser energy on (a) average ion energy, (b) peak of the TOF signal and (c) total 256 charge. The error bars represented the standard deviation derived from the data of five experiments.

257 In order to further compare the plasma characteristics between the foam and solid 258 Ni plasmas, the effect of the laser energy on the average ion energy, peak of the TOF 
signal, and the total charge is shown in Figure 3. The average ion energy can be 260 calculated as

$$
\begin{aligned}
& \bar{E}=1 / 2 M\left(\frac{L}{\bar{t}}\right)^{2} \\
& \bar{t}=\int_{t_{i}}^{t_{f}} t V(t) d t / \int_{t_{i}}^{t_{f}} V(t) d t
\end{aligned}
$$

262 where $M$ is the mass of the ion, $t_{i}$ and $t_{f}$ respectively are the start and end time 263 values of the ion signal, $V(t)$ is the voltage measured by the FC. As expected, the 264 average ion energy of the solid and foam Ni plasma increased with the increase of laser 265 energy, but the rising rate was slowly decreasing, and that of foam Ni plasma showed a 266 saturation trend. The average ion energy of solid Ni plasma was greater than that of 267 foam Ni plasma and the difference gradually increased with the increase of laser energy. 268 For instance, the difference of the average ion energy between solid and foam Ni plasma 269 was $536.4 \mathrm{eV}$ at $100 \mathrm{~mJ}$, and this value became $1498.7 \mathrm{eV}$ at $400 \mathrm{~mJ}$. These results 270 implies that ions are thermalizing at lower kinetic energy and weak laser energy 271 dependence in case of foam targets which is very important for the fusion research and 272 EUV debris reducing where lower energy ions are preferable.

273 It is well known that the laser can only propagate in the plasma below the critical 274 electron density. During the interaction between laser and plasma, an ionization wave 275 and a rarefaction wave will be created in the conduction zone and the interface between 276 the plasma and vacuum, respectively ${ }^{20,23}$. If the velocity of ionization wave propagating 277 into the bulk target is higher than that of sound in the already heated matter, significant 278 hydrodynamic motion will not be generated in spite of pressure gradients are very large 279 at the position of heat front ${ }^{20}$. Various studies ${ }^{20,23,30}$ related to laser produced under280 dense target (the ionized electron density lower than the laser critical density) plasma 281 pointed out that the ionization wave generated by laser ablating under-dense target is 282 supersonic and the rarefaction wave is subsonic. In the case of under-dense plasma, no 283 hydrodynamic motion of the dense plasma occurs because of the supersonic laser 284 heating, resulting in no hydrodynamic shock wave generated. Therefore, the absorbed 
285 laser energy will not be transferred to hydrodynamic motion. Alternatively, a 286 hydrodynamic shock wave will be created inside the over-dense plasma plume which 287 enhances the conversion of the laser energy to the ion kinetic energy ${ }^{20,36,40}$. The electron 288 density $n_{e}$ of the target can be calculated from the mass density $\rho$ by ${ }^{41}$

$$
n_{e}=\rho \frac{\bar{Z}}{M_{\text {atom }}} A_{v}
$$

where $\bar{Z}$ is the average ionization state, $M_{\text {atom }}$ is the average atomic weight, and $A_{v}$ is the Avogadro's number. Although the electron density of the foam and solid Ni under absolute ionization $\left(2.56 \times 10^{24} \mathrm{~cm}^{-3}\right.$ for solid $\mathrm{Ni}, 1.72 \times 10^{23} \mathrm{~cm}^{-3}$ for foam $\left.\mathrm{Ni}\right)$ were both greater than the critical density $\left(1.03 \times 10^{21} \mathrm{~cm}^{-3}\right.$ for $1.064 \mu \mathrm{m}$ laser $)$, the hydrodynamic shock wave formation was suppressed due to the low initial density of foam Ni to a certain extent, thereby reducing the conversion of laser energy to ion kinetic energy for foam Ni.

Figure 3(b) and Figure 3(c) show the peak of the TOF signal and the total charge under different laser energies, respectively. The peak of the TOF signal reflects the maximum ion density of plasma plume in a specific direction and the total charge represents the ion yield during the interaction between the laser and targets. It is obvious that the two parameters of the solid $\mathrm{Ni}$ were greater than those of the foam $\mathrm{Ni}$ due to the huge difference in target density. The total charge was calculated by integrating the corresponding TOF signal shown in Figure $2^{42}$ :

$$
Q=\int_{t_{i}}^{t_{f}} \frac{V(t) d t}{R}
$$

where $R$ is the load resistance. It can be seen that the peak of the TOF signal and total charge increased with laser energies for foam and solid Ni. The peak of the TOF signal

307 for foam and soldi $\mathrm{Ni}$ both increased about 2 times in the studied laser energy range.

308 The rise of these two parameters with laser energies can be due to the following two 309 reasons. First, laser with high-energy would ablate more target material and have a 310 higher mass ablation rate. Second, the increased laser energy enhanced the plasma 
311 heating process by the IB mechanism and lead to a higher degree of ionization in the 312 plasma ${ }^{42,43}$. By comparing the peak of TOF signal between the foam and solid Ni, it 313 can be clearly seen that the peak of TOF signal of the solid Ni was about 4 times that 314 of the foam Ni under each laser energy, which confirms that the maximum ion density 315 in solid Ni plasma plume was much higher than that of foam Ni plasma. The total charge 316 in Figure 3(c) also shows a saturation behavior for solid Ni in the studied laser energy 317 range, which was not obvious for foam Ni. The saturation behavior for solid Ni could 318 be ascribed to the more intense plasma shielding and reflection at the critical density 319 point, which was also verified by the mass ablation rate shown in section 3.3. Similar 320 saturation trend was found in laser ablation of other solid materials ${ }^{38,44}$. The reason of 321 the difference between the solid and foam Ni plasma could be explained as follows. 322 During the processes of nanosecond laser target interactions, once the critical density 323 is reached, the rest of the laser energy will be reflected and absorbed at the edge of the 324 plasma plume which is then used to increase the ion kinetic ${ }^{45}$. Generally, the radiation 325 absorption length along the beam axis can be treated as the so-called geometrical transparency length $L_{T}$, which is determined by the classical collision mechanisms $20,36,40$ :

$$
L_{T}=\frac{9.2 \times 10^{-8}}{Z}\left(\frac{A}{Z}\right)^{2} \frac{T^{3 / 2}}{\lambda^{2} \rho^{2}}
$$

where $Z$ is the charge of the plasma ions, $A$ is the atomic number, $T$ is the electron temperature $(\mathrm{keV}), \lambda$ is the wavelength of the laser $(\mu \mathrm{m})$, and $\rho$ is the plasma

331 density $\left(\mathrm{g} / \mathrm{cm}^{3}\right)$. From Equation (5), it is clear that the geometrical transparency length

332 for laser radiation is inversely proportional to the squared foam (plasma) density, which 333 means that the geometrical transparency length is shorter for solid Ni target due to its 334 higher initial density. Therefore, when the high-power laser irradiated the solid Ni target, 335 the plasma generated by the leading edge of the laser pulse would reach the critical 336 electron density faster than that of the foam target and most part of the absorbed laser 337 energy only took place near the critical electron density point, resulting in a higher 
338 electron density gradient. Main part of the increased laser energy will be reflected at 339 the critical density point in the case of the solid $\mathrm{Ni}$, which prevented to ablate more 340 target material. Due to the lower initial density of the foam Ni, the critical density point 341 was deeper inside of the target and the laser can penetrate the target to a much greater 342 depth ${ }^{20,33}$. In other words, the under-dense corona zone (the electron density lower than 343 critical density) of the foam Ni plasma was larger than that of solid Ni plasma and the 344 electron temperature and density in the foam Ni plasma plume were more uniform ${ }^{22}$. 345 This phenomenon can also be reflected to a certain extent from the lower and wider 346 TOF signal of the foam Ni shown in Figure 2. These characteristics of foam Ni 347 exhibited the so-called volumetric heating effect and a higher laser energy absorption 348 as the laser energy increased ${ }^{20,22,23,33}$. Therefore, the saturation behavior of total charge 349 was more obvious for solid $\mathrm{Ni}$. The increase of the total charge for foam Ni plasma was 350 much greater than that of solid Ni plasma in the studied laser energy range. 

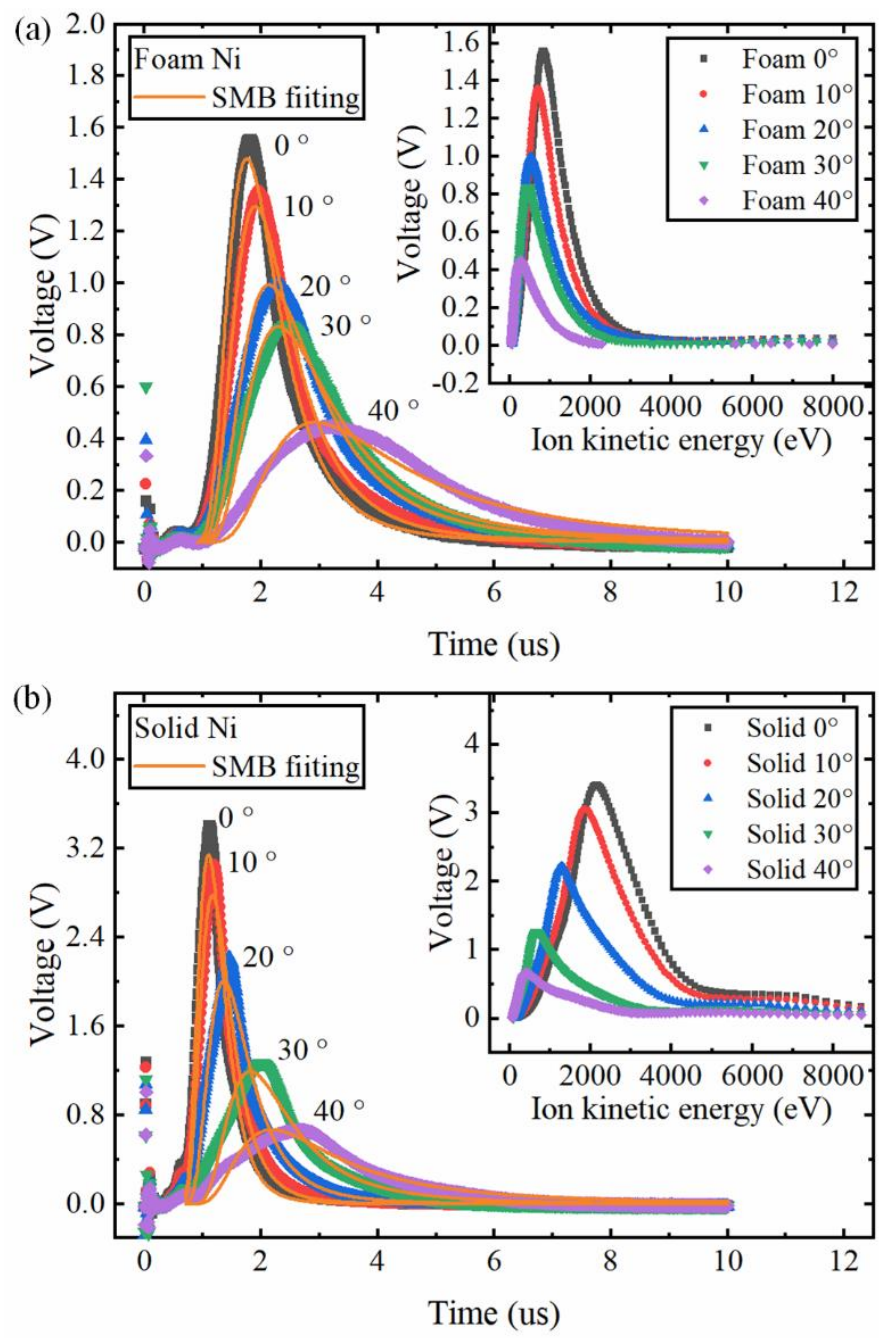

Figure 4 Typical ion TOF signal obtained from Faraday cup ion collector for various angular (a) Foam $\mathrm{Ni}$ and (b) Solid Ni. The $0^{\circ}$ means that the FC and the laser spot were on the same horizontal plane. The Shifted Maxwell Boltzmann distribution was used to fit the thermal peaks. The inset shows the ion kinetic energy distribution converted from the TOF signal.

Understanding the angularly resolved distributions of the ion flux and kinetic energies are essential to study the mechanisms of ion production and plume expansion dynamics. For better understanding of the angular dependence of the ions emitted from foam and solid Ni plasma, the FC was mounted on a rotating handle with an angle meter and placed at various angles with respect to the horizontal plane, and the ion TOF

361 signals were recorded and shown in Figure 4 when the laser energy was $400 \mathrm{~mJ}$. The 362 solid lines given in Figure 4 correspond to the SMB fitting using Equation (1). It is clear 363 that the peak of the TOF signals for solid and foam Ni occurred at later times and the 364 amplitudes decreased with the angles. The TOF signal amplitude of the solid Ni was 365 larger than that of the foam Ni at various angles and the TOF signal of the solid Ni had 
366 a narrower distribution than that of the foam Ni. The inset in Figure 4 shows the ion 367 kinetic energy distribution and the results illustrated that the maximum probable energy 368 of the ions from both foam and solid Ni plasma decreased with an increase in off-normal 369 angles. When the off-normal angles increased from $0^{\circ}$ to $40^{\circ}$, the most probable ion 370 kinetic energy of the solid Ni plasma dropped from $2120 \mathrm{eV}$ to $400 \mathrm{eV}$, and that of foam 371 Ni plasma dropped from $830 \mathrm{eV}$ to $270 \mathrm{eV}$. 

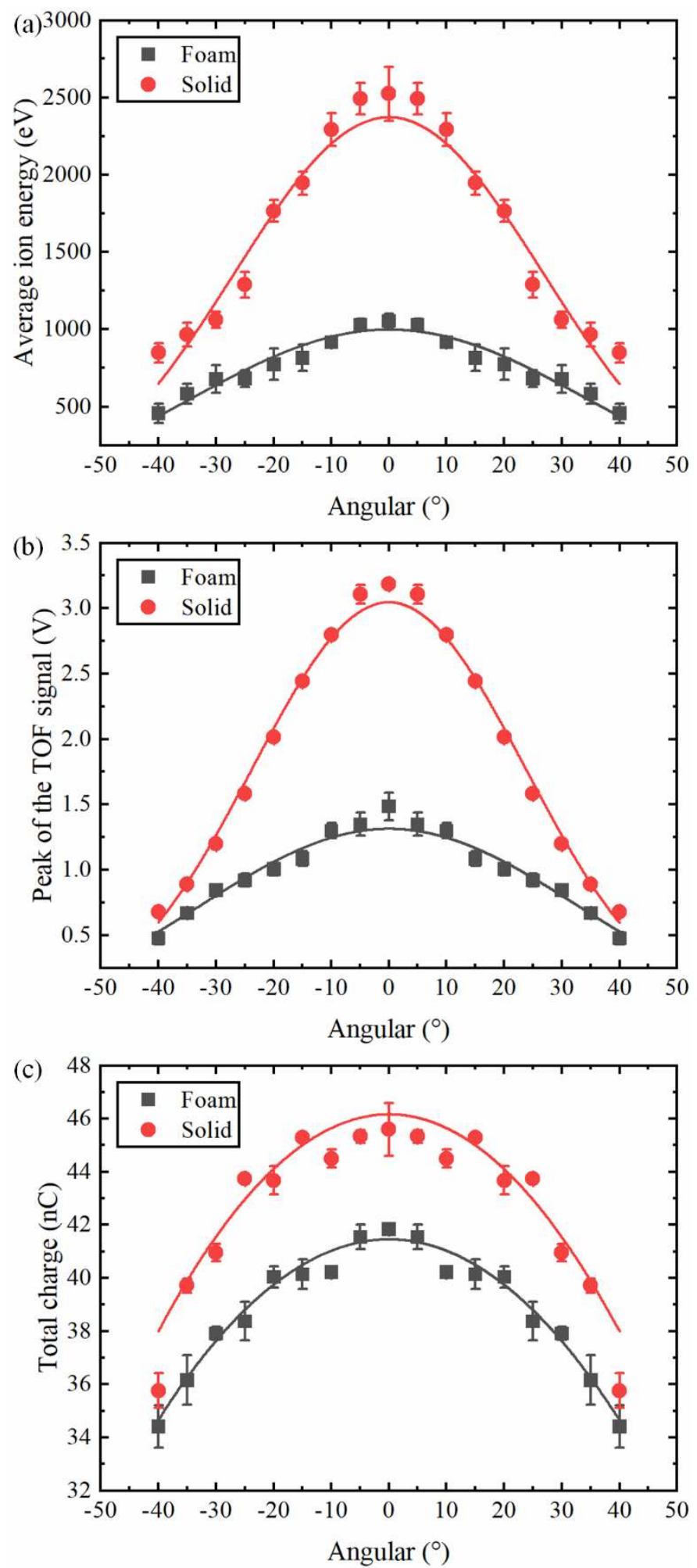

Figure 5 Angular dependence of (a) average ion energy, (b) peak of the TOF signal and (c) total charge.

374 The error bars represented the standard deviation derived from the data of five experiments. The solid 375 curves in each plot represent the $\cos ^{n} \theta$ fitting to the various data sets.

376 In order to compare the angular dependence of the ions between the solid and foam 377 Ni plasma, the average ion energy, peak of the TOF signal and total charge under 
different angles were calculated and shown in Figure 5. The TOF signals were recorded for positive angles but copied to their negative angles for comparison assuming hemispherical expansion of the plasma plume. Generally, the angular distribution of the ions can be described by the $\cos ^{n} \theta$ function when a laser pulse is irradiated on a planar target in the normal direction ${ }^{37}$, where $n$ is typically larger than 1 . The solid curves shown in Figure 5 represent the $\cos ^{n} \theta$ fitting to the various data sets. The results show that the average ion energy, signal amplitudes, and the total charge strongly peaked when the angle was directly normal to the target and this behavior was more obvious for solid Ni plasma.

These three ion parameters of solid Ni were larger than that of foam Ni at various angles, but the difference between them gradually decreased with the increase of the angle. The average ion energy and peak of the TOF signal of solid Ni showed a stronger angular dependence than that of foam Ni. For example, the average ion energy and peak of the TOF signal of solid Ni were reduced to only $33.6 \%$ and $21.3 \%$ of the initial values at $40^{\circ}$, respectively. In the case of foam $\mathrm{Ni}$, the average ion energy and peak of the TOF signal were reduced to $43.4 \%$ and $32.2 \%$ of the initial values at the same angle, respectively. Based on the fitting results, the exponent $n$ was estimated with an accuracy of $10 \%$ at 3.09 and 4.87 for foam and solid Ni in Figure 5(a), and at 3.41 and 6.11 in Figure 5(b), respectively. These results indicated that the plasma plume expansion was more isotropic for foam $\mathrm{Ni}$ compared with solid $\mathrm{Ni}$, which could be ascribe to the larger geometrical transparency length and the volumetric heating effect. In the case of the total charge, both the solid Ni plasma and foam Ni plasma have similar angular distributions. Although the average ion energy and peak of the TOF signal of foam Ni plasma were much lower than that of solid Ni plasma, the total charge between the foam Ni target and solid Ni target was comparable in spite of the density of the foam Ni target was less than 1/10th of the solid Ni target. These results indicated that a large number of ions were coming from the large volume of foam Ni plasma and supported the more volumetric absorption of laser energy for low-density foam targets, which was 
406 also consistent with the laser produced low-density foam gold ${ }^{29}, \mathrm{SiO}_{2}{ }^{46}$ and polymer

407 aerogels ${ }^{36}$ plasma studies and some simulations ${ }^{30,33}$.

$408 \quad 3.2$ Plasma plumes

409 While the FC ion collector can provide interesting ion dynamic results along with 410 the specific expansion direction, it is difficult to provide the two-dimensional shape of 411 the plasma plume. In order to further investigate the plasma expansion dynamics, fast 412 photography studies of foam Ni plasma and solid Ni plasma were carried out in order 413 to record a series of time-resolved plasma plume images. These images provide the 414 details of the LPP expansion dynamics, especially at very early stages after plasma 415 generation. 


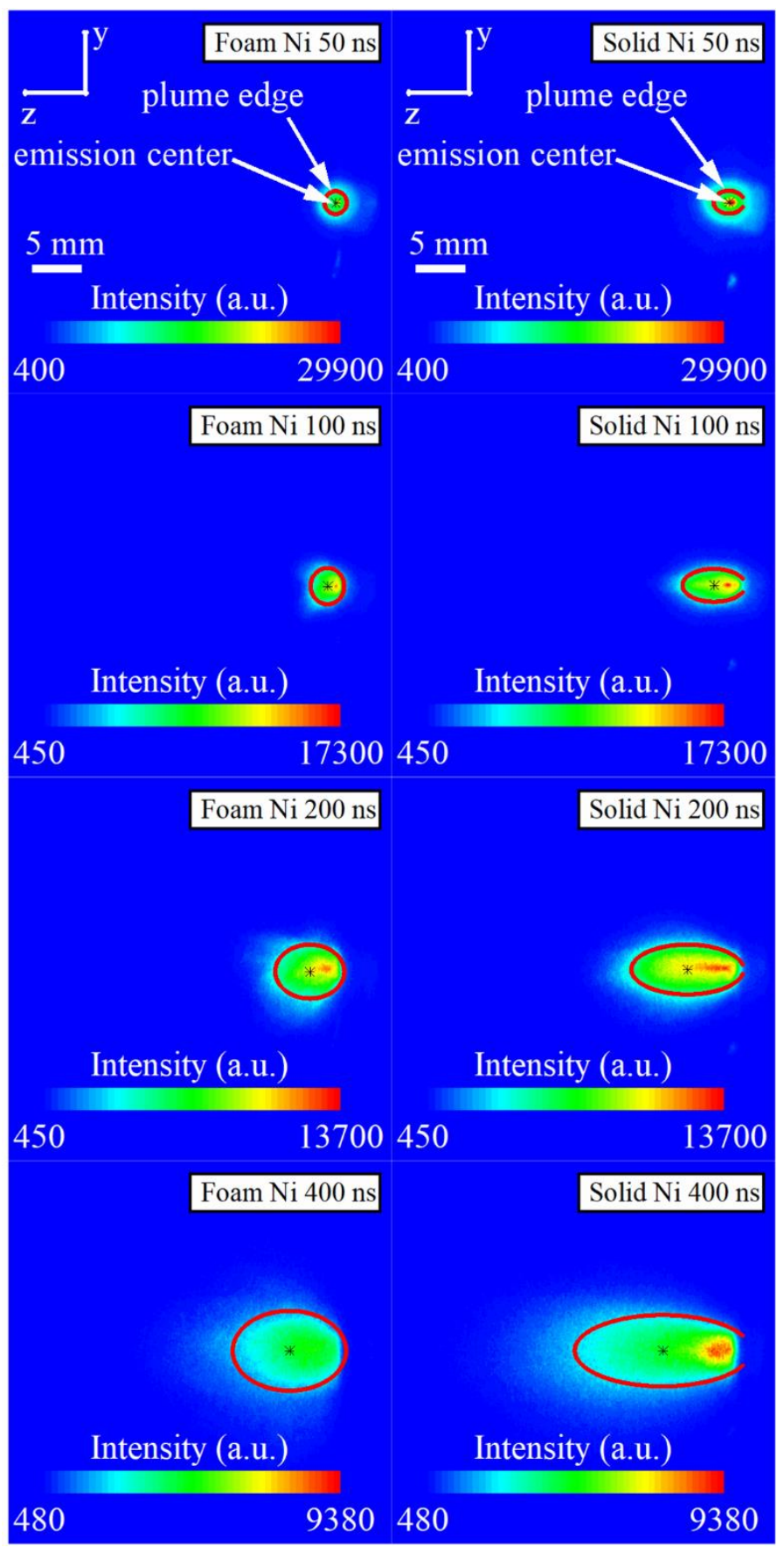

Figure 6 Time sequence of the two-dimensional ICCD images showing the spatial-temporal evolution of 418 the plasma plume for foam and solid Ni. The laser irradiated on the targets along the $\mathrm{z}$ direction and its 419 energy was $100 \mathrm{~mJ}$. The black star $\left(^{*}\right)$ in each image represents the emission center determined by the 420 1st-order moment of the emission intensity. The red ellipse in each image represents the plasma plume 421 edge determined by the 2nd-order moment of the emission intensity. Each image at particular time is 422 normalized to maximum intensity with respect to solid $\mathrm{Ni}$ images.

423 Figure 6 gives the time sequence of the two-dimensional ICCD images showing 424 the spatial-temporal evolution of the plasma plume for the foam and solid Ni. The laser 425 energy used for this measurement was $100 \mathrm{~mJ}$ and the gate width of the ICCD was fixed 
to $5 \mathrm{~ns}$ at early times ( $<100 \mathrm{~ns})$ to minimize the spatial-temporal mixing and to optimize the imaging of internal structures. After $100 \mathrm{~ns}$, the gate width was fixed to $10 \mathrm{~ns}$ to compensate for lower radiation at later times. Unlike the plasma plume expanding into an ambient gas condition, a sharp boundary was not observed in ICCD imaged plumes. In order to further investigate the plume expansion dynamics, determining the emission center and plume edge is very important and necessary. Based on the magnetohydrodynamics and self-similar expansion model ${ }^{47}$, the plasma plume generated by a laser irradiating a plane target can be approximated as an ellipsoid. Therefore, the plume shape of the LPP can be suitably described by an elliptic equation. In this study, the 1st-order moment and 2nd-order moment of the plume emission intensity were adopted to define the emission center and plume edge, respectively. The emission center was calculated as ${ }^{48}$ :

$$
\bar{z}=\frac{\int_{-\infty}^{+\infty} \int_{-\infty}^{+\infty} z I(z, y) d z d y}{\int_{-\infty}^{+\infty} \int_{-\infty}^{+\infty} I(z, y) d z d y}, \bar{y}=\frac{\int_{-\infty}^{+\infty} \int_{-\infty}^{+\infty} y I(z, y) d y d z}{\int_{-\infty}^{+\infty} \int_{-\infty}^{+\infty} I(z, y) d y d z}
$$

where $z$ is the direction along the laser irradiating, $y$ is the direction parallel to the target surface, and $I$ is the emission intensity of the plume. The semi-major axis and semi-minor axis of plume edge ellipse were calculated as ${ }^{48}$ :

$$
\begin{aligned}
& R_{z}=2 \sqrt{\sigma_{z}^{2}}=2 \sqrt{\frac{\int_{-\infty}^{+\infty} \int_{-\infty}^{+\infty}(z-\bar{z})^{2} I(z, y) d z d y}{\int_{-\infty}^{+\infty} \int_{-\infty}^{+\infty} I(z, y) d z d y}} \\
& R_{y}=2 \sqrt{\sigma_{y}^{2}}=2 \sqrt{\frac{\int_{-\infty}^{+\infty} \int_{-\infty}^{+\infty}(y-\bar{y})^{2} I(z, y) d z d y}{\int_{-\infty}^{+\infty} \int_{-\infty}^{+\infty} I(z, y) d z d y}}
\end{aligned}
$$

Generally, the plasma plume of foam and solid Ni both showed an almost symmetrical distribution with respect to the normal direction of the targets, and both expanded freely with little external viscous force because of the lower ambient pressure and the much higher initial pressure of the plasma. It is clear that the emission intensity of solid $\mathrm{Ni}$ was higher than that of foam $\mathrm{Ni}$ due to the higher initial density. The plume shape of the solid Ni plasma appeared as an oblong ellipse at each time, while that of foam $\mathrm{Ni}$ 
449 plasma tended to be more circular, especially at early times. By comparing the position 450 of plume edge, it can be seen that the expansion velocity of solid Ni plasma was much 451 higher than that of foam Ni plasma along the laser irradiating direction. This meant that 452 the expansion dynamics of solid Ni plasma was more anisotropic than that of foam $\mathrm{Ni}$ 453 plasma, which was consistent with the FC signal studies.
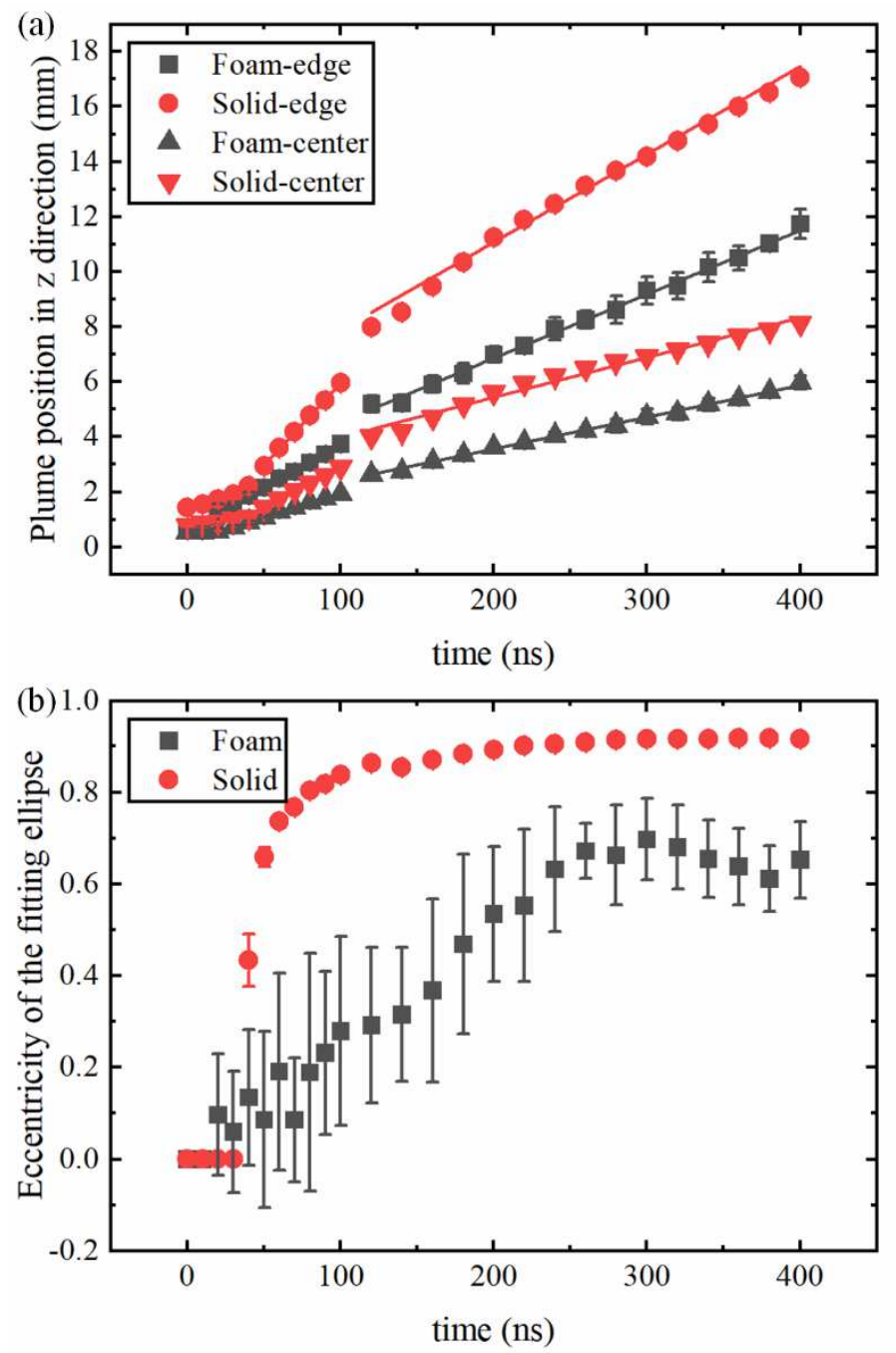

Figure 7 (a) Temporal variation of the plume position in $z$ direction obtained from ICCD images. The 'edge' $(\boldsymbol{\bullet}, \bullet)$ represents the position of fitting ellipse edge. The 'center' $(\boldsymbol{\Lambda}, \boldsymbol{\nabla})$ represents the position of the fitting ellipse center. The solid lines correspond to the linear fit. (b)Temporal variation of the eccentricity of the fitting ellipse. The error bars represented the standard deviation derived from the data of five experiments.

$460 \quad$ Figure 7 gives a more direct comparison of the plume position along the laser 461 irradiating direction between the foam and solid Ni plasma as well as the eccentricity 462 of the fitting ellipse. The results show a linear trend for the plasma plume of foam and 
463

464

465

466

467

468

469

470

471

472

473

474

475

476

477

478

479

480

481

482

483

484

485

solid $\mathrm{Ni}$ at later times which means that the plasma was expanding freely because the ambient conditions were almost near vacuum. This linear trend was more obvious for later times due to the less collisions. There was a jump in plume position between 100 ns and $120 \mathrm{~ns}$ because the gate width of ICCD was fixed from $5 \mathrm{~ns}$ to $10 \mathrm{~ns}$. The average velocities of the plume edge derived from the plot for foam and solid Ni were $2.94 \times 10^{6}$ $\mathrm{cm} / \mathrm{s}$ and $4.27 \times 10^{6} \mathrm{~cm} / \mathrm{s}$, and that of the emission center for foam and solid Ni were $1.49 \times 10^{6} \mathrm{~cm} / \mathrm{s}$ and $2.03 \times 10^{6} \mathrm{~cm} / \mathrm{s}$, respectively. These results were comparable with the average velocity $\left(3.45 \times 10^{6} \mathrm{~cm} / \mathrm{s}\right.$ for foam Ni, $5.43 \times 10^{6} \mathrm{~cm} / \mathrm{s}$ for solid $\left.\mathrm{Ni}\right)$ and center-of-mass velocity $\left(1.79 \times 10^{6} \mathrm{~cm} / \mathrm{s}\right.$ for foam $\mathrm{Ni}, 4.65 \times 10^{6} \mathrm{~cm} / \mathrm{s}$ for solid $\left.\mathrm{Ni}\right)$ determined from the FC signal. The velocities derived from the ICCD images were smaller than that determined from FC signal and the reason could be as follows. The TOF signal recorded by Faraday cup was only contributed by ions in the plume. However, the plasma plume captured by the ICCD was recorded integrally from a wavelength range of $180-850 \mathrm{~nm}$, which meant that the plume intensity included much radiation from neutrals. Nevertheless, according to the electrostatic model ${ }^{37}$, the neutrals cannot be accelerated in the self-electrostatic field and their velocities are much lower than ions in the plasma plume, which has been demonstrated by theoretical and experimental studies ${ }^{37,49}$. Therefore, the neutrals emission included in the plume would reduce the average velocity determined from the time resolved ICCD images.

Figure 7(b) shows the temporal variation of the eccentricity of the fitting ellipse. The eccentricity of the fitting ellipse could reflect the shape of the plume and be determined as:

$$
e=\sqrt{1-\left(\frac{R_{y}}{R_{z}}\right)^{2}}
$$

where $R_{y}$ and $R_{z}$ are the semi-minor axis and semi-major axis of plume edge ellipse. The eccentricity of foam and solid Ni plasma plume was at 0 at very early times which meant that plasma plume shape was more like a circle, and then the eccentricity increased with time until a plateau was reached. The eccentricity of solid Ni plasma 
plume had a much higher increasing rate than that of foam Ni plasma plume and the 491 final value of solid Ni plasma plume was higher than that of foam plasma plume. The results indicated that the plume shape gradually evolved from a circle to a flatter ellipse

493 with time and the solid Ni plasma had a stronger angular dependence than that of foam

494 Ni plasma. The larger eccentricity jitter of the foam Ni plasma plume could be due to 495 the fact that the foam Ni target had a random network structure and was kept moving during the experiment, resulting in a slightly pressure difference in the plume produced 497 by each laser shot. The eccentricity jitter of the foam Ni plasma plume was smaller at 498 later times since the plume difference was compensated with plume expansion.

$499 \quad 3.3$ Mass ablation rate

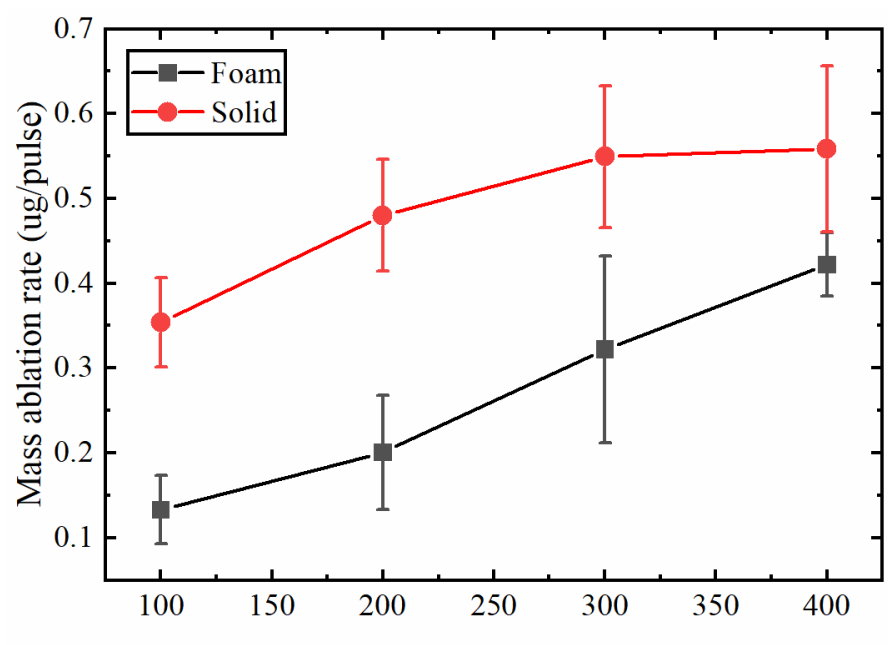

500

501

502

503

504

505

506

507

508

Laser energy $(\mathrm{mJ})$

Figure 8 Trend of mass ablation rate for foam and solid Ni targets at various laser energies. The error bars represented the standard deviation derived from the data of five experiments.

Studying the mass ablation rate gives useful information about the interaction between laser and target. Figure 8 shows the trend of mass ablation rate for foam and solid Ni targets at various laser energies. The mass ablation rate of solid and foam $\mathrm{Ni}$ increased about 1.6 times and 3.2 times in the investigated laser energy range, respectively. Although the mass ablation rate of soldi Ni was higher than that of foam $\mathrm{Ni}$ at various laser energy, the values between them were somewhat comparable in spite of the huge difference in target density. In addition, a near saturation trend of mass ablation rate was found for the solid $\mathrm{Ni}$ which was absent for foam $\mathrm{Ni}$ in the investigated laser energy range. These results were consistent with the total charge shown in Figure 
$5123(\mathrm{c})$ and indicated that the plasma shielding effect was more intense for solid Ni.

\section{4. Conclusion}

514 Foam targets are expected to be more efficient candidates than solid targets for 515 laser produced plasma (LPP) for extreme ultraviolet (EUV) and x-ray radiation sources

516 due to the expected plasma conditions that can be optimized regarding plasma opacities, 517 volumetrics heating effects, and the produced ions debris characteristics. The ion 518 emission from solid and foam Ni targets during $1064 \mathrm{~nm}$ wavelength Nd:YAG laser 519 ablation was investigated in detail using Faraday cup (FC). The average ion energy, 520 peak of the time-of-flight (TOF) signal, and the total charge was measured as a function 521 of laser energy and off-normal angles. It was shown that the TOF signal of foam Ni 522 plasma demonstrated a lower and wider distribution than that of solid Ni plasma on the 523 time scale, which indicated that the ion density distribution in the foam Ni plasma 524 plume was relatively more uniform compared to solid Ni plasma. The ion emission 525 features at various angles showed a stronger angular dependence for solid Ni plasma. 526 The average ion energy and peak of the TOF signal of foam Ni plasma were much lower 527 than that of solid Ni plasma, but the total charge was comparable in spite of the huge 528 difference in target density. These results supported the more volumetric absorption of 529 laser energy in case of low-density foam targets due to the larger volume of the foam 530 plasma below the critical density of the laser beam. The plasma plume images recorded 531 by an intensified charged coupled device (ICCD) camera illustrated that the plasma 532 plume of solid Ni plasma was more elongated along the laser irradiating direction 533 compared with that of foam Ni plasma. The average velocities determined from time534 resolved images was slightly smaller than the average velocities derived from FC 535 signals, which was ascribe to the low-speed vapor and atom emission contained in 536 plume. The mass ablation rate of solid Ni was higher than that of foam Ni, but the 537 difference between them decreased with the increase of the laser energy, which 538 indicated that the plasma shielding effect was more intense for solid Ni. The kinetic 539 energy of ions produced from the foam Ni target was much lower than that of the solid 
540 Ni target. This is very important in terms of the lifetime of the optical collecting system,

541 e.g., in nanolithography devices. Lower energy ions of the foam targets will cause much

542 less damage than the energetic ions of the solid targets, therefore much longer lifetime.

\section{Acknowledgements}

$544 \quad \mathrm{Y}$. Wu and X. Wang thanks the support from Basic and Applied Basic Research 545 Major Program of Guangdong Province (No.2019B030302003). The authors would 546 like to acknowledge the support and the help in conducting the experiments at the 547 Center for Materials Under Extreme Environment (CMUXE) and Davidson School of 548 Chemical Engineering, Purdue University, USA. Y. Wu also thanks the financial 549 support from the China Scholarship Council (CSC) (No. 201906160071) and the 550 experimental facilities support from Prof. Vilas Pol, Mr. Tong Xu and Mr. Zheng Li.

\section{Conflict of Interest}

552 The authors declare no conflict of interest.

\section{Author Contributions}

$554 \quad$ Yaoxing Wu designed experiments and wrote the original manuscript, Girik Jain 555 helped the experiments and checked the English, Tatyana Sizyuk revised the original 556 manuscript, Xinbing Wang provided some experimental methods and revised the 557 original manuscript, Ahmed Hassanein provided the conceptions and supervised the 558 experiments and revised the original manuscript.

\section{References}

560 1. Freeman, J. R., Harilal, S. S. \& Hassanein, A. Enhancements of extreme ultraviolet 561 emission using prepulsed Sn laser-produced plasmas for advanced lithography 562 applications. J. Appl. Phys. 110, 083303 (2011).

563 2. Back, C. A. et al. Efficient Multi-keV Underdense Laser-Produced Plasma 564 Radiators. Phys. Rev. Lett. 87, 275003 (2001).

565 3. Betti, R. \& Hurricane, O. A. Inertial-confinement fusion with lasers. Nat. Phys. 12, 
567 4. Mendys, A. et al. Investigations of laser-induced plasma in argon by Thomson 568 scattering. Spectrochim. Acta Part B At. Spectrosc. 66, 691-697 (2011).

569 5. Bailey, J. E. et al. Experimental investigation of opacity models for stellar interior, 570 inertial fusion, and high energy density plasmas. Phys. Plasmas 16, 058101 (2009).

571 6. Antonelli, L. et al. Laser-driven shock waves studied by x-ray radiography. Phys. 572 Rev. E 95, 063205 (2017).

573 7. Sizyuk, T. Consequences of high-frequency operation on EUV source efficiency. 574 Phys. Plasmas 24, 083105 (2017).

575 8. Sizyuk, T. Characterization of radiative and kinetic properties of laser produced 576 plasmas for efficient extreme ultraviolet nanolithography sources. J. Appl. Phys. 126, $577163302(2019)$.

578 9. Sizyuk, V., Sizyuk, T., Hassanein, A. \& Johnson, K. Recycling of laser and plasma 579 radiation energy for enhancement of extreme ultraviolet sources for nanolithography. 580 J. Appl. Phys. 123, 013302 (2018).

581 10. Kasperczuk, A. et al. Influence of the focal point position on the properties of a 582 laser-produced plasma. Phys. Plasmas 14, 102706 (2007).

583 11. Sizyuk, V., Hassanein, A. \& Sizyuk, T. Three-dimensional simulation of laser584 produced plasma for extreme ultraviolet lithography applications. J. Appl. Phys. 100, $585103106(2006)$.

586 12. Sizyuk, T. \& Hassanein, A. Tuning laser wavelength and pulse duration to improve 
587 the conversion efficiency and performance of EUV sources for nanolithography. Phys.

588 Plasmas 27, 103507 (2020).

589 13. Jones, O. S. et al. Experimental and calculational investigation of laser-heated 590 additive manufactured foams. Phys. Plasmas 28, 022709 (2021).

591 14. Harilal, S. S., Sizyuk, T., Sizyuk, V. \& Hassanein, A. Efficient laser-produced 592 plasma extreme ultraviolet sources using grooved Sn targets. Appl. Phys. Lett. 96, $593111503(2010)$.

594 15. Sizyuk, T. \& Hassanein, A. Optimum pre-pulsing and target geometry of LPP for 595 efficient EUV and BEUV sources. in Extreme Ultraviolet (EUV) Lithography VI (eds.

596 Wood, O. R. \& Panning, E. M.) vol. 942294220 G (SPIE, 2015).

597 16. Hassanein, A. Effects of plasma spatial profile on conversion efficiency of laser598 produced plasma sources for EUV lithography. J. Micro/Nanolithography, MEMS, 599 MOEMS 8, 041503 (2009).

600 17. Sizyuk, T. \& Hassanein, A. Optimizing laser produced plasmas for efficient 601 extreme ultraviolet and soft X-ray light sources. Phys. Plasmas 21, 083106 (2014).

602 18. Sizyuk, T. \& Hassanein, A. Comprehensive 3D simulation of laser/target 603 interactions for various applications. in 2014 IEEE 41st International Conference on 604 Plasma Sciences (ICOPS) held with 2014 IEEE International Conference on High605 Power Particle Beams (BEAMS) 1-1 (IEEE, 2014). 606 doi:10.1109/PLASMA.2014.7012476.

607 19. Jiang, K., Pukhov, A. \& Zhou, C. T. TJ $\mathrm{cm}-3$ high energy density plasma 
608 formation from intense laser-irradiated foam targets composed of disordered carbon 609 nanowires. Plasma Phys. Control. Fusion 63, 015014 (2021).

610 20. Chaurasia, S. et al. Enhancement of keV X-rays from low-density cellulose 611 triacetate (TAC) foam targets. Phys. Plasmas 24, 073110 (2017).

612 21. Sizyuk, T. \& Hassanein, A. The role of plasma evolution and photon transport in 613 optimizing future advanced lithography sources. J. Appl. Phys. 114, 083109 (2013).

614 22. Nagai, K., Musgrave, C. S. A. \& Nazarov, W. A review of low density porous 615 materials used in laser plasma experiments. Phys. Plasmas 25, 030501 (2018).

616 23. Dong, Y. et al. Efficient soft x-ray sources from laser-irradiated gold foam targets 617 with well-controlled impurities. Nucl. Fusion 58, 016038 (2018).

618 24. Sizyuk, T. \& Hassanein, A. Extending the path for efficient extreme ultraviolet 619 sources for advanced nanolithography. Phys. Plasmas 22, 093101 (2015).

620 25. Fujimoto, J. et al. Laser-produced plasma-based extreme-ultraviolet light source 621 technology for high-volume manufacturing extreme-ultraviolet lithography. $J$. 622 Micro/Nanolithography, MEMS, MOEMS 11, 021111-1 (2012).

623 26. Musgrave, C. S. A., Shoji, S. \& Nagai, K. Easy-handling minimum mass laser 624 target scaffold based on sub-millimeter air bubble -An example of laser plasma extreme 625 ultraviolet generation-. Sci. Rep. 10, 5906 (2020).

626 27. Okuno, T. et al. Low-density tin targets for efficient extreme ultraviolet light 627 emission from laser-produced plasmas. Appl. Phys. Lett. 88, 161501 (2006).

628 28. Harilal, S. S. et al. Extreme-ultraviolet spectral purity and magnetic ion debris 
629 mitigation by use of low-density tin targets. Opt. Lett. 31, 1549 (2006).

630 29. Kaur, C. et al. Demonstration of gold foam plasma as bright x-ray source and slow 631 ion emitters. Plasma Phys. Control. Fusion 61, 084001 (2019).

632 30. Yunsong, D., Lu, Z., Jiamin, Y. \& Wanli, S. Detailed energy distributions in laser633 produced plasmas of solid gold and foam gold planar targets. Phys. Plasmas 20, 123102 634 (2013).

635 31. Nagai, K., Miyamoto, K., Iyoda, T., Pan, C. \& Gu, Z. Monolithic and Low-Density 636 (<50 mg/cm 3 ) Metal Oxides Fabricated Using Electrospinning: Vanadium Oxide and 637 Copper Oxide Examples. Fusion Sci. Technol. 59, 216-220 (2011).

638 32. Tanabe, M. et al. Titanium dioxide nanofiber-cotton targets for efficient multi-keV 639 x-ray generation. Appl. Phys. Lett. 93, 051505 (2008).

640 33. Fazeli, R. Tuning laser plasma x-ray source for single shot microscopy using nano641 porous targets. Opt. Lett. 41, 5250 (2016).

642 34. Hara, H. et al. Emission of water-window soft x-rays under optically thin 643 conditions using low-density foam targets. Opt. Lett. 43, 3750 (2018).

644 35. Zhang, L. et al. Reducing wall plasma expansion with gold foam irradiated by laser. 645 Phys. Plasmas 22, 110703 (2015).

646 36. Kaur, C. et al. Interaction of high-power laser radiation with low-density polymer 647 aerogels. Quantum Electron. 47, 495-502 (2017).

648 37. Wang, X. et al. Ion kinetic energy distributions in laser-induced plasma. 649 Spectrochim. Acta Part B At. Spectrosc. 99, 101-114 (2014). 
651 electron emission from laser-produced plasma. Phys. Plasmas 20, 073114 (2013).

652 39. Hassanein, A. \& Sizyuk, T. Laser produced plasma sources for nanolithography-

653 Recent integrated simulation and benchmarking. Phys. Plasmas 20, 053105 (2013).

654 40. Yan, X., Tuo, Z., Shuanggui, L. \& Jiamin, Y. Beneficial effect of CH foam coating 655 on x-ray emission from laser-irradiated high-Z material. Phys. Plasmas 18, 053301 656 (2011).

657 41. Mariscal, D. A. et al. Laser transport and backscatter in low-density $\mathrm{SiO} 2$ and $\mathrm{Ta}$ 6582 O 5 foams. Phys. Plasmas 28, 013106 (2021).

659 42. Dogar, A. H., Ullah, S., Qayyum, H., Rehman, Z. U. \& Qayyum, A. 660 Characterization of charge and kinetic energy distribution of ions emitted during 661 nanosecond pulsed laser ablation of several metals. J. Phys. D. Appl. Phys. 50, 385602 662 (2017).

663 43. Ilyas, B., Dogar, A. H., Ullah, S. \& Qayyum, A. Laser fluence effects on ion 664 emission from a laser-generated Cu plasma. J. Phys. D. Appl. Phys. 44, 295202 (2011). 665 44. Claeyssens, F., Lade, R. J., Rosser, K. N. \& Ashfold, M. N. R. Investigations of 666 the plume accompanying pulsed ultraviolet laser ablation of graphite in vacuum. J. Appl. 667 Phys. 89, 697-709 (2001).

668 45. Amoruso, S., Armenante, M., Berardi, V., Bruzzese, R. \& Spinelli, N. Absorption 669 and saturation mechanisms in aluminium laser ablated plasmas. Appl. Phys. A Mater. 670 Sci. Process. 65, 265-271 (1997). 
671 46. Kaur, C. et al. X-ray and ion emission studies from subnanosecond laser-irradiated 672 SiO 2 aerogel foam targets. Laser Part. Beams 35, 505-512 (2017).

673 47. Doggett, B. \& Lunney, J. G. Expansion dynamics of laser produced plasma. J. Appl. 674 Phys. 109, 093304 (2011).

675 48. Chen, Z., Wang, X., Zuo, D., Lu, P. \& Wang, J. Investigation of Nd:YAG laser 676 produced tin droplet plasma expansion. Laser Phys. Lett. 13, 056002 (2016).

677 49. Wu, Y., Sizyuk, T., Termini, N. C., Wang, X. \& Hassanein, A. Spectroscopic 678 studies of laser produced Bi-Pb alloy plasma. Spectrochim. Acta Part B At. Spectrosc. 679 175, $106033(2021)$.

680 
Figures

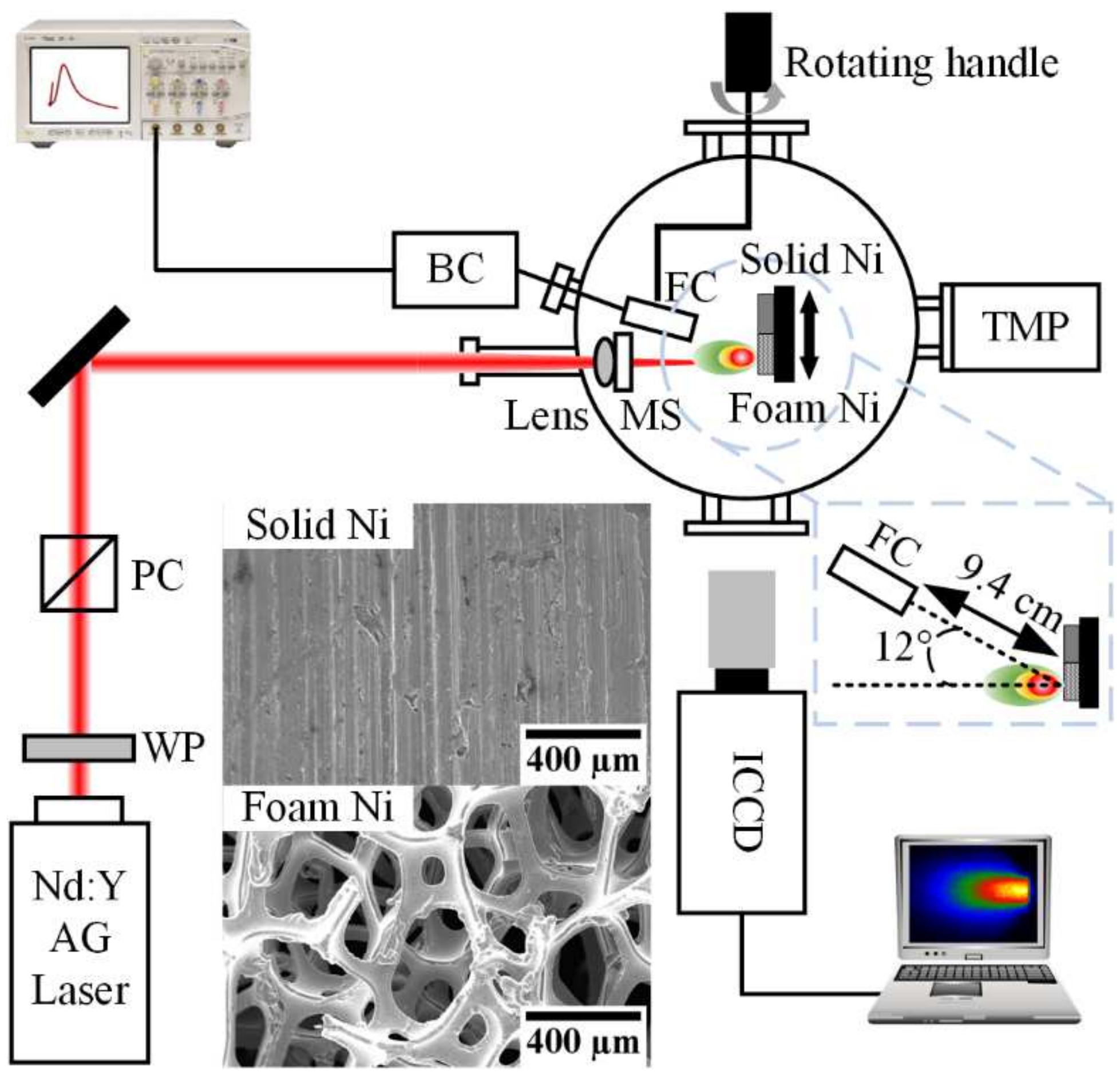

Figure 1

The schematic of the experimental setup in CMUXE and the pictures of solid and foam Ni under SEM. BC, biasing circuit; FC, Faraday cup; MS, microscope slide, TMP, turbomolecular pump; PC, polarizing cube; WP, half waveplate; ICCD, intensified charged coupled device. 

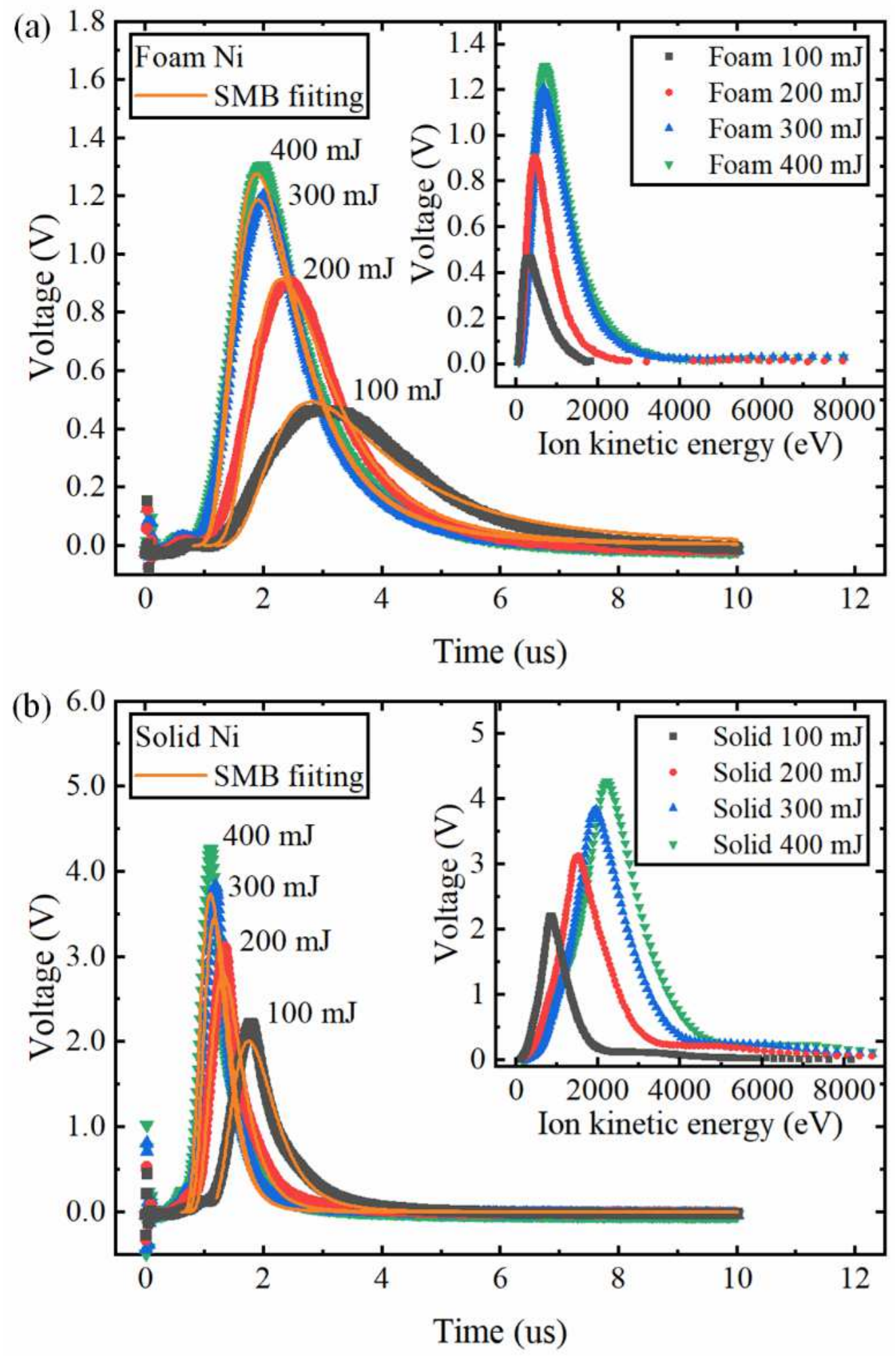

Figure 2

Typical ion time-of-flight (TOF) signal obtained from the Faraday cup ion collector for various laser energies for (a) Foam Ni and (b) Solid Ni. The Shifted Maxwell Boltzmann distribution was used to fit the thermal peaks. The inset shows the ion kinetic energy distribution converted from the TOF signal. 

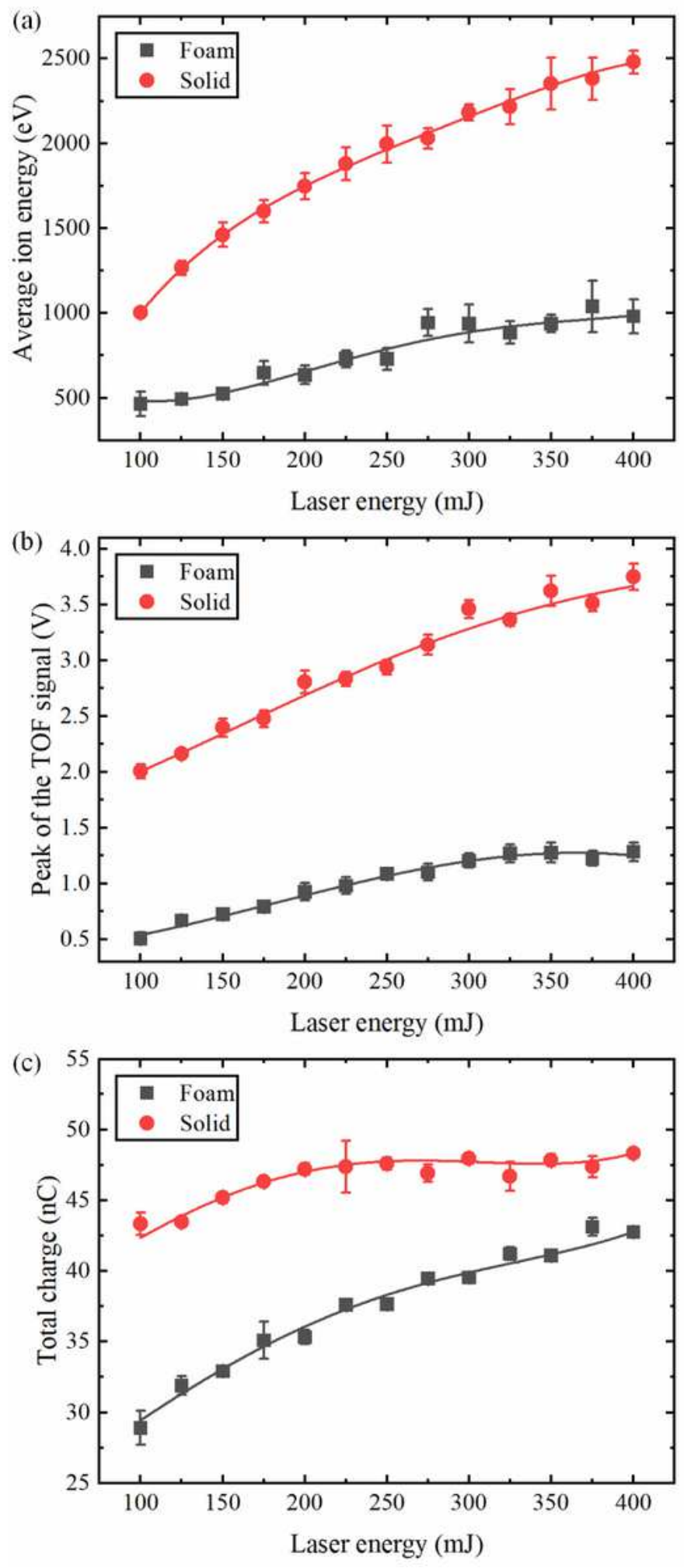

\section{Figure 3}

Effect of the laser energy on (a) average ion energy, (b) peak of the TOF signal and (c) total charge. The error bars represented the standard deviation derived from the data of five experiments. 

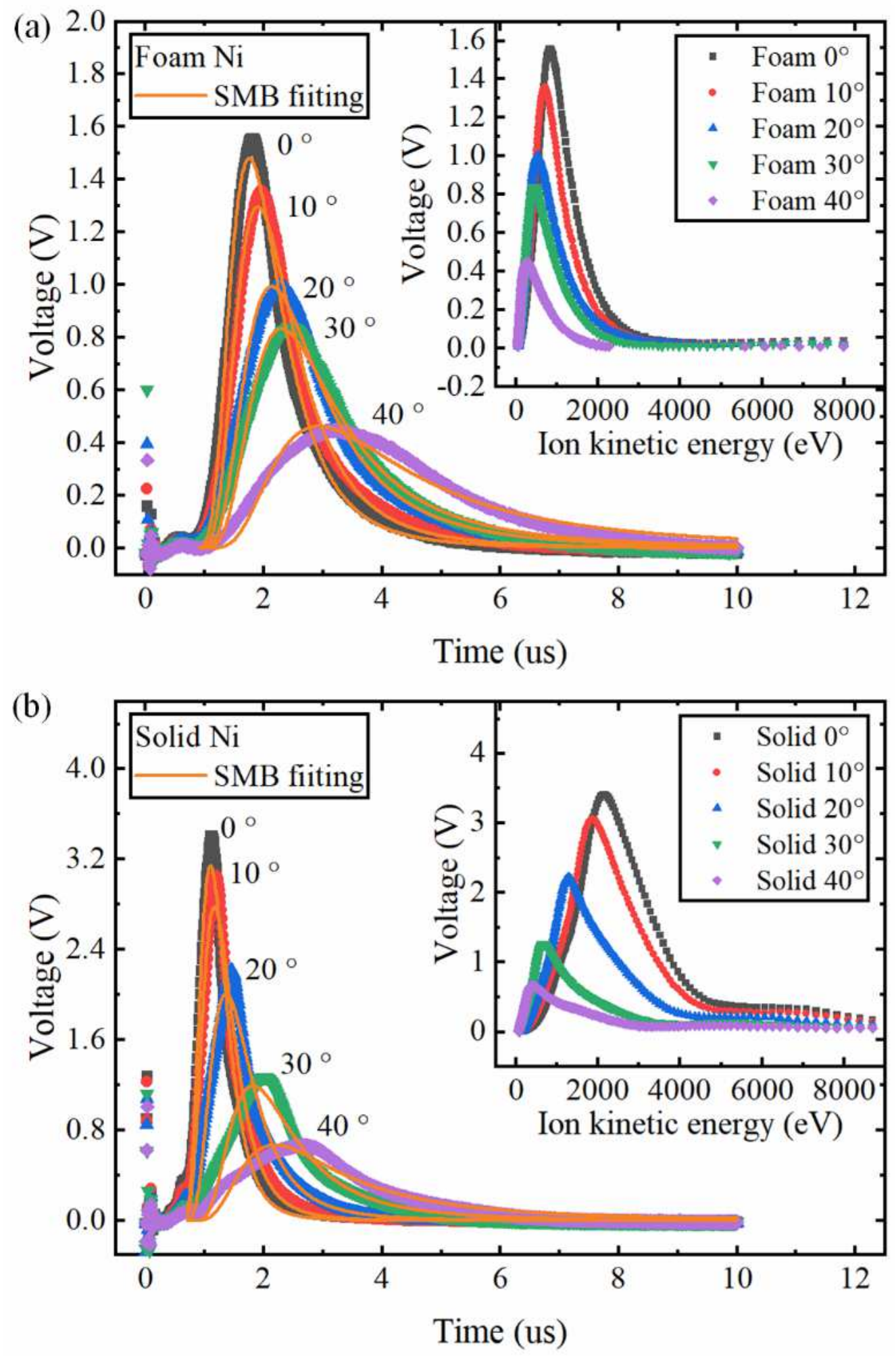

\section{Figure 4}

Typical ion TOF signal obtained from Faraday cup ion collector for various angular (a) Foam Ni and (b) Solid $\mathrm{Ni}$. The $0^{\circ}$ means that the FC and the laser spot were on the same horizontal plane. The Shifted Maxwell Boltzmann distribution was used to fit the thermal peaks. The inset shows the ion kinetic energy distribution converted from the TOF signal. 

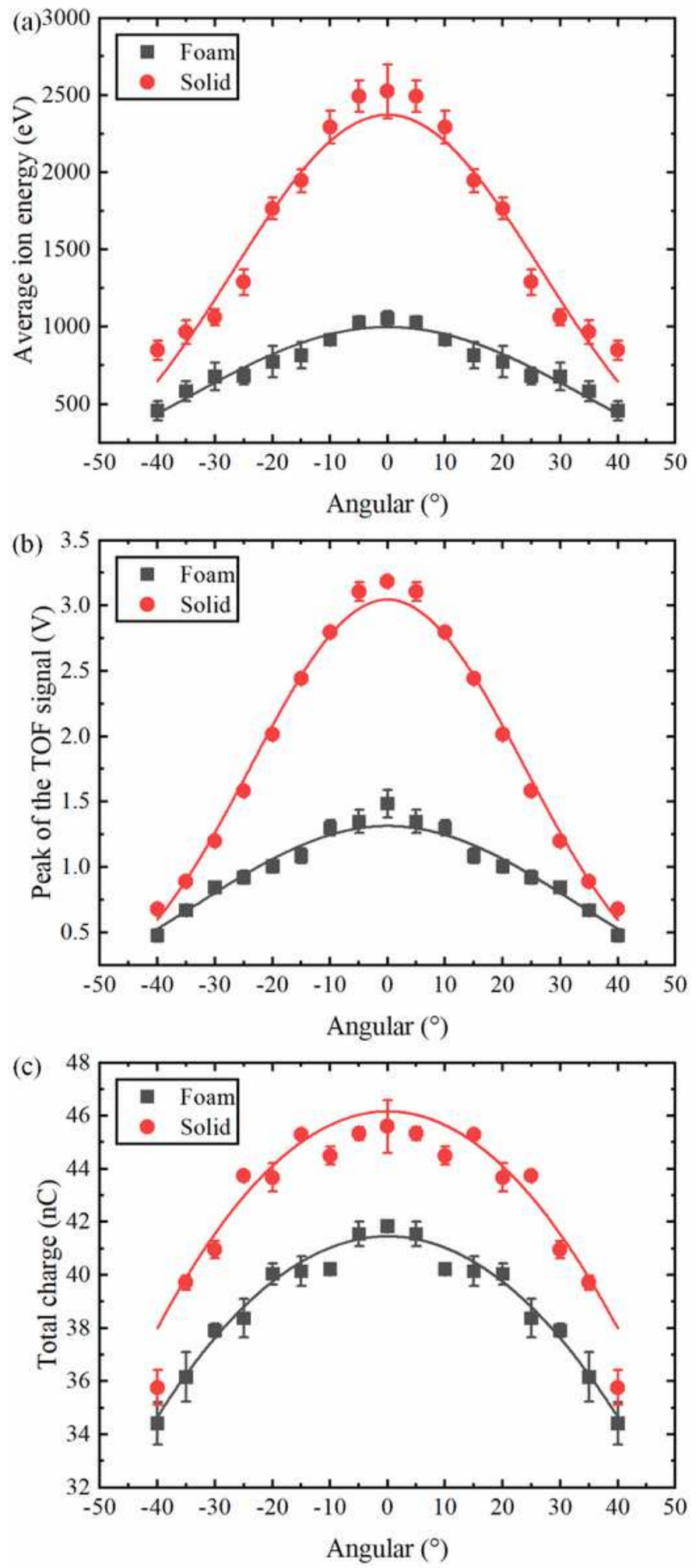

\section{Figure 5}

Angular dependence of (a) average ion energy, (b) peak of the TOF signal and (c) total charge. The error bars represented the standard deviation derived from the data of five experiments. The solid curves in each plot represent the $\cos ^{\wedge} n \theta$ fitting to the various data sets. 


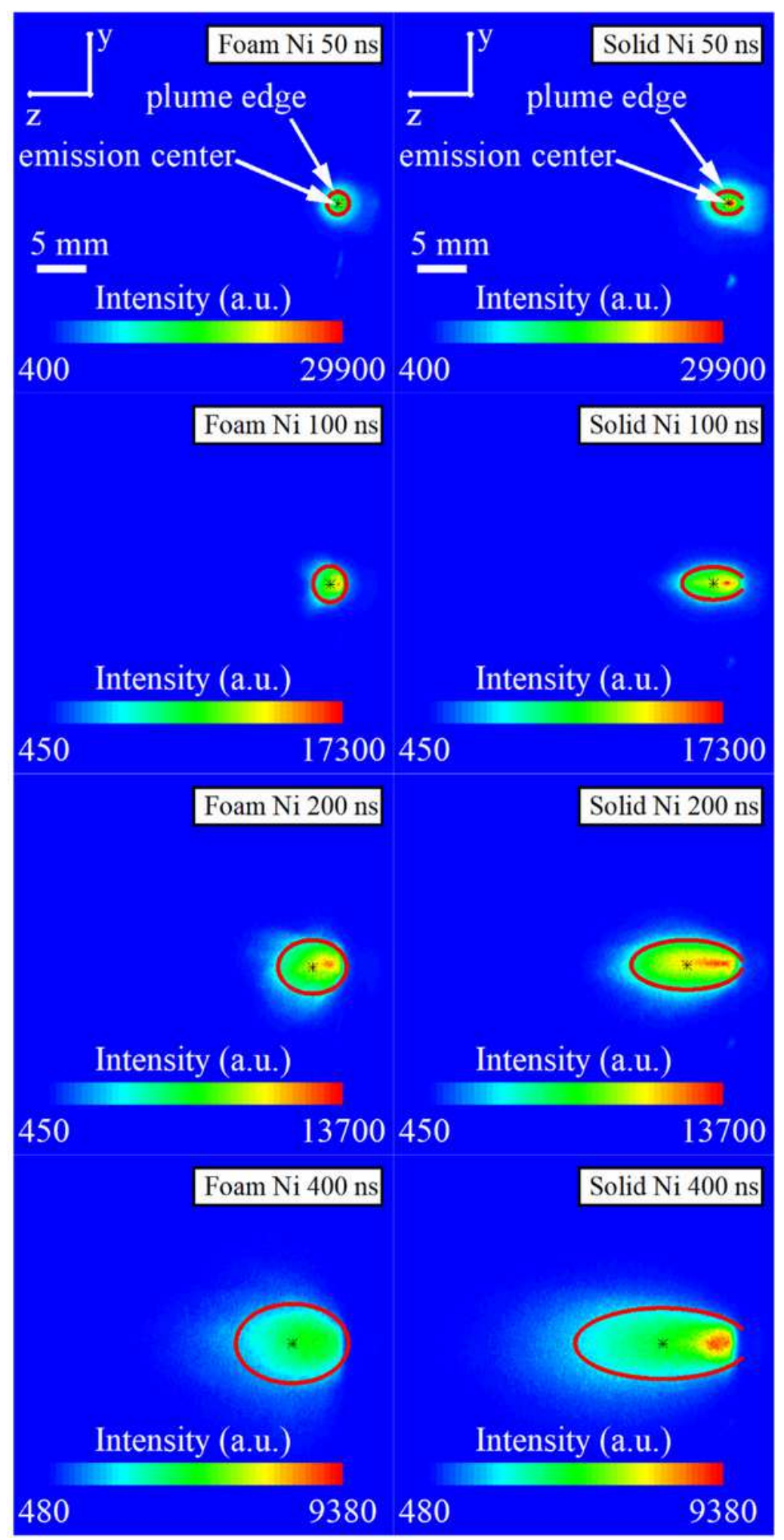

\section{Figure 6}

Time sequence of the two-dimensional ICCD images showing the spatial-temporal evolution of the plasma plume for foam and solid $\mathrm{Ni}$. The laser irradiated on the targets along the $\mathrm{z}$ direction and its energy was $100 \mathrm{~mJ}$. The black star (*) in each image represents the emission center determined by the 1 st-order moment of the emission intensity. The red ellipse in each image represents the plasma plume 
edge determined by the 2nd-order moment of the emission intensity. Each image at particular time is normalized to maximum intensity with respect to solid Ni images.
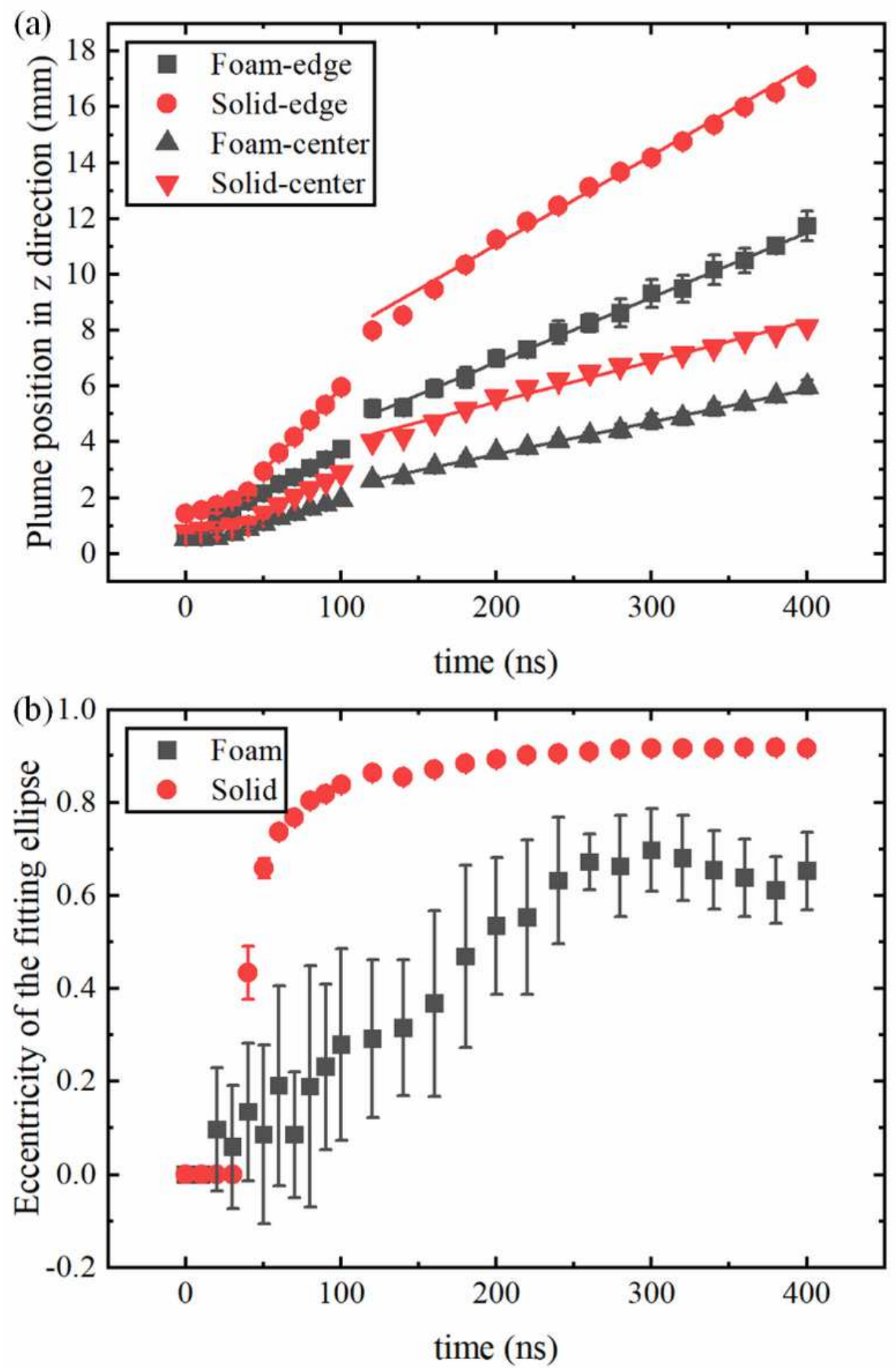

Figure 7



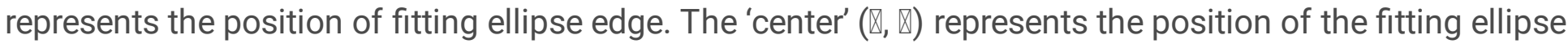


center. The solid lines correspond to the linear fit. (b)Temporal variation of the eccentricity of the fitting ellipse. The error bars represented the standard deviation derived from the data of five experiments.

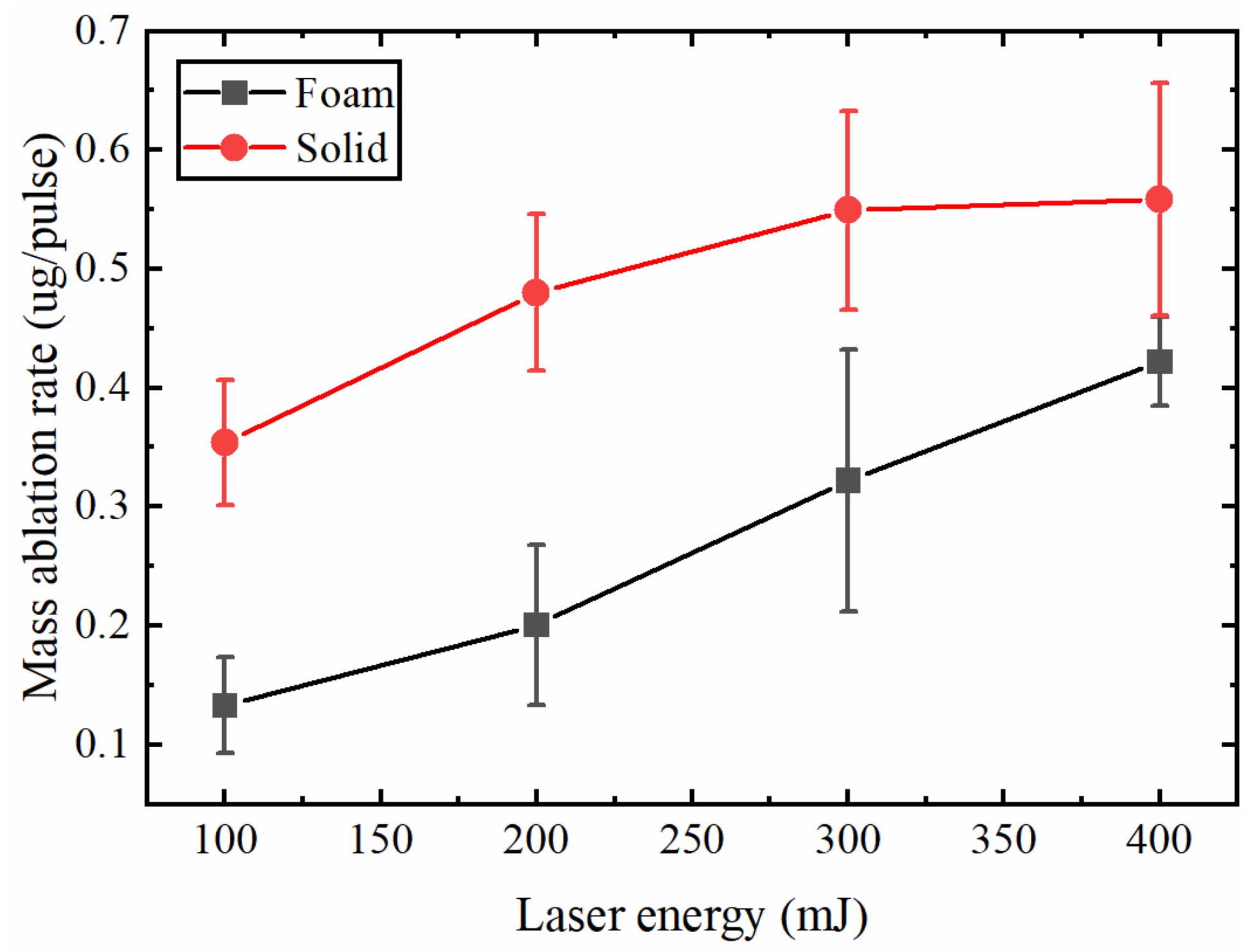

Figure 8

Trend of mass ablation rate for foam and solid Ni targets at various laser energies. The error bars represented the standard deviation derived from the data of five experiments. 